\title{
Energetics of dislocation transformations in hep metals
}

\author{
Zhaoxuan $\mathrm{Wu}^{\mathrm{a}, \mathrm{b}, *}$, Binglun Yin ${ }^{\mathrm{a}}$, W. A. Curtin ${ }^{\mathrm{a}}$ \\ ${ }^{a}$ Institute of Mechanical Engineering, École Polytechnique Fédérale de Lausanne, Lausanne \\ CH-1015, Switzerland \\ ${ }^{b}$ Institute of High Performance Computing, 1 Fusionopolis Way, \#16-16, Connexis, Singapore \\ 138632, Singapore
}

\begin{abstract}
Dislocation core structures of hcp metals are highly complex and differ significantly among the hcp family. Some dislocations undergo unconventional transformations that have significant effects on the material plastic flow. Here, the energetics of dislocation dissociations are analyzed in a general anisotropic linear elastic theory framework for transformations in which changes in the partial Burgers vectors are small. Quantitative analyses on various transformations are made using DFT-computed stacking fault energies and partial Burgers vectors. Specifically, possible transformations of the mixed, edge, and screw $\langle\mathbf{c}+\mathbf{a}\rangle$ and screw $\langle\mathbf{a}\rangle$ dislocations in $6 \mathrm{hcp}$ metals $(\mathrm{Mg}, \mathrm{Ti}, \mathrm{Zr}, \mathrm{Re}, \mathrm{Zn}, \mathrm{Cd})$ are studied. Climb dissociation of mixed or edge $\langle\mathbf{c}+\mathbf{a}\rangle$ dislocations to the Basal plane is energetically favorable in all 6 metals and thus only limited by thermal activation. The $\langle\mathbf{c}+\mathbf{a}\rangle$ screw dislocation is energetically preferable on Pyramidal I for Ti, $\mathrm{Zr}$, and $\mathrm{Re}$, and on Pyramidal II for $\mathrm{Zn}$ and $\mathrm{Cd}$. In Mg, the energy difference between screw $\langle\mathbf{c}+\mathbf{a}\rangle$ on Pyramidal I and II planes is small, suggesting relatively easy cross-slip. For the screw $\langle\mathbf{a}\rangle$, Basal dissociation is energetically favorable in $\mathrm{Mg}$, $\mathrm{Re}, \mathrm{Zn}$ and $\mathrm{Cd}$, while Prism dissociation is strongly favorable in Ti and $\mathrm{Zr}$. Only $\mathrm{Ti}, \mathrm{Zr}$ and Re show a metastable state for dissociation on the Prism plane, and the energy difference between screw $\langle\mathbf{a}\rangle$ on the Prism and Pyramidal I planes is relatively small in all systems, suggesting relatively easy cross-slip of $\langle\mathbf{a}\rangle$ in Ti and Zr. The elastic analysis thus provides a single framework able to capture the controlling energetics for different dissociations and slip systems in hcp metals. When the calculated energy differences are very small, the results point to the need for detailed modeling of the atomistic core structure. Moreover, the analyses ratio-
\end{abstract}

\footnotetext{
${ }^{*}$ Corresponding author

Email address: zhaoxuan.wu@epfl.ch (Zhaoxuan Wu)
} 
nalize broad experimental observations on dominant slip systems and dislocation behaviours, and provide predictions for possible transformations for the family of hcp metals.

Keywords: HCP, Dislocations, Dissociation, Elastic Energy, Stacking Fault Energy

\section{Introduction}

In crystalline metals, dislocations are the primary carriers of plastic deformation, with plastic strain generated by the motion of dislocation cores as driven by applied stresses [1]. Dislocation cores, i.e. the line defects that terminate atomic slip, can dissociate and/or transform into different local atomic structures while preserving the macroscopic topological character of the Burgers vector $\mathbf{b}$ and line direction $\boldsymbol{\xi}$. Dislocations with the same $\mathbf{b}$ and $\boldsymbol{\xi}$ but different core structures can have drastically different behaviours in response to applied stresses, which can lead to anomalous yield/flow behaviour in metals. One well-known example is the Kear-Wilsdorf (KW) lock [2] where $\langle 10 \overline{1}\rangle$ dislocations on $\{111\}$ planes move onto $\{010\}$ planes in certain intermetallic compounds of $\mathrm{L} 1_{2}$ structure $[3,4]$. More recently, in situ transmission electron microscopy (TEM) experiments and $a b$ initio calculations [5] show that the jerky glide (locking-unlocking) of $\langle\mathbf{a}\rangle$ dislocation in hexagonal close-packed (hcp) Ti is associated with a core transition between Prism and Pyramidal (Pyr.) I planes. The KW lock and locking-unlocking phenomena in Ti are special cases because both are driven by cross-slip of screw dislocations onto atomically different planes. Core structure transitions of edge or mixed dislocations are less common, and unexpected according to conventional dislocation theory. Surprisingly, recent molecular dynamics (MD) simulations in hcp Mg show that edge and mixed $\langle\mathbf{c}+\mathbf{a}\rangle$ dislocations on easy-glide Pyr. II and I planes can climb-dissociate into effectively sessile partials oriented on the Basal plane $[6,7]$. These $\langle\mathbf{c}+\mathbf{a}\rangle$ transitions are distinctly different mechanism from the KW lock, and their consequences on macroscopic material properties are also starkly different in practical terms. The KW lock provides strengthening and creep-resistance at high temperatures for a class of commercial superalloys [4], whereas the transformation from easy glide to Basal-dissociated $\langle\mathbf{c}+\mathbf{a}\rangle$ dislocation undesirably hardens the crystallographic $\langle\mathbf{c}\rangle$ direction of hcp metals such as $\operatorname{Mg}[6]$.

While the $\langle\mathbf{a}\rangle$ transition has only been demonstrated in $\mathrm{Ti}$ and the $\langle\mathbf{c}+\mathbf{a}\rangle$ transitions have only been shown in MD simulations of $\mathrm{Mg}$, such transitions (par- 
ticularly the $\langle\mathbf{c}+\mathbf{a}\rangle$ transitions ) may be ubiquitous among hcp metals, as indicated by various experiments in $\mathrm{Ti}$ [8], $\mathrm{Zr}$ [9], Ti-6.6Al [10] and Ti-36.5at.\% Al [11]. It is thus desirable to examine the energetics of possible $\langle\mathbf{a}\rangle$ and $\langle\mathbf{c}+\mathbf{a}\rangle$ transitions across the family of hcp metals. Direct atomistic simulations would be useful, but are not currently feasible due to high computational costs in density functional theory (DFT) calculations and/or lack of well-validated interatomic potentials for MD simulations of most hep metals. On the other hand, linear elastic theory $[12,13,14]$ combined with experimental elastic constants and DFT-computed stacking fault (SF) energies and Burgers vectors provides an approximate general framework for analysing the energetics of dislocation dissociations, and that is the direction taken in this work. Specifically, we present a detailed analysis of the energetics of dislocation dissociations in a wide range of hcp metals $(\mathrm{Mg}, \mathrm{Ti}$, $\mathrm{Zr}, \mathrm{Re}, \mathrm{Zn}$ and Cd) using new DFT-based SF energies and Burgers vectors of the dissociated partials, and anisotropic linear elastic theory [12, 13, 14]. In cases where the core energies of the dislocation partials are expected to be comparable, we determine the most stable dislocation configurations and identify the primary slip systems for screw $\langle\mathbf{a}\rangle$ and $\langle\mathbf{c}+\mathbf{a}\rangle$ dislocations. For both the edge and mixed $\langle\mathbf{c}+\mathbf{a}\rangle$ dislocations dissociated on Pyr. II and I planes, our results show that a climb-dissociated $\langle\mathbf{c}+\mathbf{a}\rangle$ transition onto the Basal plane is energetically favorable, due to the strong angular term in the elastic interactions when the climb distance exceeds $\sim 2-3 \mathrm{~nm}$. Therefore, such $\langle\mathbf{c}+\mathbf{a}\rangle$ dislocation transitions are highly likely a generic feature of hcp metals. For the $\langle\mathbf{c}+\mathbf{a}\rangle$ screw dislocations on Pyr. I and II planes, the SF energies play the dominant major role in determining the favorable dissociation plane, with dissociated $\langle\mathbf{c}+\mathbf{a}\rangle$ screw dislocations quite favorable on Pyr. I planes in $\mathrm{Ti}, \mathrm{Zr}$ and $\mathrm{Re}$ and on Pyr. II planes in $\mathrm{Zn}$ and $\mathrm{Cd}$. For $\mathrm{Mg}$, the Pyr. I and II SF energies are comparable and the theory predicts the screw $\langle\mathbf{c}+\mathbf{a}\rangle$ dislocation to have a slightly lower energy ( $\sim 3-30 \mathrm{meV} / \AA)$ on the Pyr. II plane than on the Pyr. I plane. For the screw $\langle\mathbf{a}\rangle$ dislocations, Basal dissociation is energetically favorable in $\mathrm{Mg}, \mathrm{Re}, \mathrm{Zn}$ and $\mathrm{Cd}$, while Prism dissociation is strongly favored in $\mathrm{Ti}$ and $\mathrm{Zr}$. Comparing the $\langle\mathbf{a}\rangle$ dissociations on Prism and Pyr. I planes shows that the total energy differences are relatively small. When energy differences are small, precise results will require detailed atomistic-level analysis. However, small energy differences also imply that applied stresses and/or solid solution alloying could change the dislocation core energies and/or SF energies sufficiently to switch stable structures to the other glide plane, which may provide opportunities to optimize dislocation cross-slip and plastic flow in hcp metals [15]. Overall, our results are consistent with available atomistic simulations and broad experimental observations. The analysis, based on anisotropic elasticity and data 
from DFT calculations, is applicable to dislocation dissociations in general and offers physical insights into the major dislocation slip systems in the family of hcp metals.

In the following sections, we first review the necessary anisotropic elastic theory and analysis of the present class of problems. The energetic comparisons of different dissociations are then provided in Section 3 and our results are further discussed in Section 4.

\section{Linear elastic analysis}

Our objective is to compute the energy of a single straight dislocation with total Burgers vector $\mathrm{b}$ and line direction $\boldsymbol{\xi}$ for two different dissociated structures, $\mathbf{b}=\mathbf{b}_{1}+\mathrm{SF}+\mathbf{b}_{2}$ and $\mathbf{b}=\mathbf{b}_{1}^{\prime}+\mathrm{SF}^{\prime}+\mathbf{b}_{2}^{\prime}$, as shown in Fig. 1a and $\mathbf{b}$, and to compare these energies to establish the energetically-favorable configuration. Such an elastic analysis should be useful when $\mathbf{b}_{1} \approx \mathbf{b}_{1}^{\prime}, \mathbf{b}_{2} \approx \mathbf{b}_{2}^{\prime}$, for which changes in dislocation core energies are small, and the separation between the partials is large compared to their core radii.

For a given dissociation, we take the total energy of the dissociated dislocation as the sum of (1) the core energies $E_{\mathrm{c}}$ of each partial dislocation, (2) an elastic energy consisting of the self-energy $E_{\mathrm{e}}$ of each partial dislocation and the elastic interaction energy $\left(W_{\mathrm{e} 12}\right)$ between the two partials, and (3) the SF energy $\left(E_{\mathrm{sf}}\right)$ in between the partials,

$$
E_{\mathrm{tot}}=\left(E_{\mathrm{c} 1}+E_{\mathrm{c} 2}\right)+\left(E_{\mathrm{e} 1}+E_{\mathrm{e} 2}+W_{\mathrm{e} 12}\right)+E_{\mathrm{sf}}
$$

The Burgers vectors of the partials are determined by the positions of fully-relaxed metastable SF on the respective generalized SF $(\gamma)$ surfaces [16]. The stacking fault energy $E_{\mathrm{sf}}$ is $\gamma_{\mathrm{sf}} r_{12}$, where $\gamma_{\mathrm{sf}}$ and $r_{12}$ are the metastable SF energy and the separation between the partial cores. The dislocation elastic and interaction energies can be calculated based on the anisotropic elastic theory of straight dislocations. The elastic theory and general approach are well-established in literature $[1,12,13,17,14,18]$, but for completeness and clarity we briefly present the results needed here.

\subsection{Elastic energy of a single straight dislocation}

We use a Cartesian coordinate system $x_{i}$ and adopt a summation convention where repeated Roman indices imply summation and a comma denotes differentiation with respect to the coordinate variable $x_{i}$. Summation over Greek indices is 

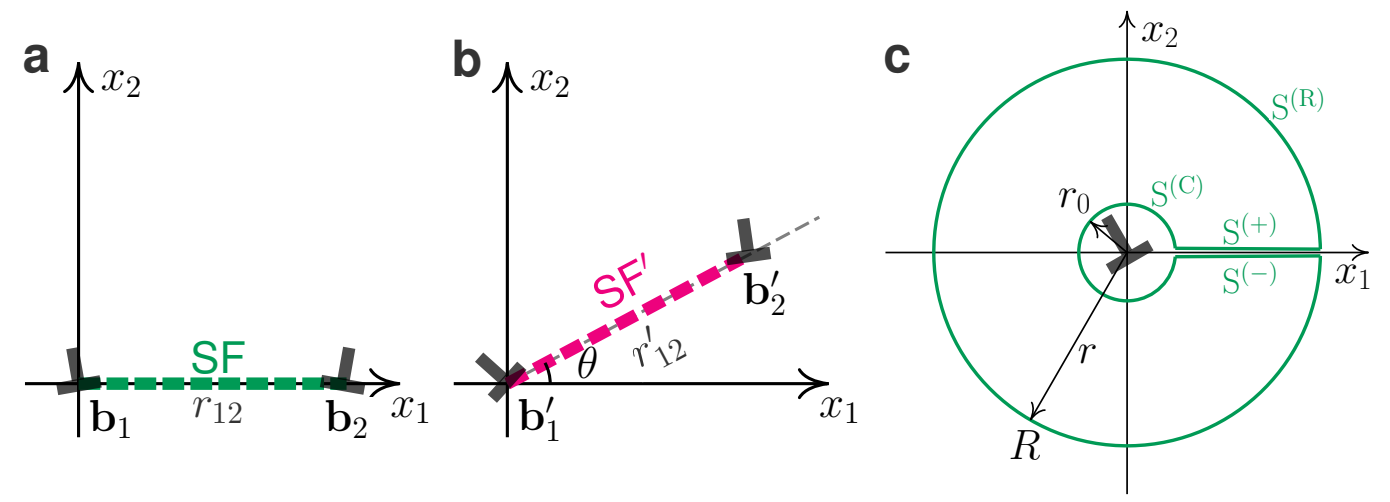

Figure 1: A dislocation with Burgers vector $\mathbf{b}$ and line direction $\boldsymbol{\xi}$ in $x_{3}$ can dissociate into (a) $\mathbf{b}_{1}+\mathrm{SF}+\mathbf{b}_{2}$ and (b) $\mathbf{b}_{1}^{\prime}+\mathrm{SF}^{\prime}+\mathbf{b}_{2}^{\prime}$. (c) A straight dislocation with Burgers vector $\mathbf{b}$ and line direction $\boldsymbol{\xi}$ along the $x_{3}$-axis at the origin is created by a cut-displace-paste procedure; $r_{0}$ and $R$ are the inner and outer radii of the cylindrical domain within which the energy is computed.

not assumed and will be written explicitly if needed. We denote the components of the displacement, stress and infinitesimal strain as $u_{i}, \sigma_{i j}$ and $\epsilon_{i j}=\left(u_{i, j}+u_{j, i}\right) / 2$, respectively. Without loss of generality, we consider an infinite straight dislocation by making a cut on the $x_{1} x_{3}$ plane between $\infty>x_{1}>0$, displacing the upper and lower cut planes by $\mathbf{b}$. This process creates a dislocation at the origin with Burgers vector $\mathbf{b}$ and line direction along the $x_{3}$-axis, as shown in Fig. 1c. We emphasize that the cut plane is not necessarily the glide plane of the dislocation. The boundary conditions of the elastic field of the dislocation at origin are

$$
\mathbf{u}\left(x_{1},+0\right)-\mathbf{u}\left(x_{1},-0\right)= \begin{cases}\mathbf{b}, & \text { for } x_{1}>0 \\ 0, & \text { for } x_{1} \leq 0\end{cases}
$$

The general solution satisfying the above boundary conditions is

$$
\begin{aligned}
& \mathbf{u}=\frac{1}{\pi} \operatorname{Im}\left\{\mathbf{A}\left\langle f\left(z_{\alpha}\right)\right\rangle \mathbf{B}^{\mathrm{T}}\right\} \mathbf{b} \\
& \boldsymbol{\psi}=\frac{1}{\pi} \operatorname{Im}\left\{\mathbf{B}\left\langle f\left(z_{\alpha}\right)\right\rangle \mathbf{B}^{\mathrm{T}}\right\} \mathbf{b}
\end{aligned}
$$

where

$$
\begin{aligned}
\left\langle f\left(z_{\alpha}\right)\right\rangle & =\operatorname{diag}\left[f\left(z_{1}\right), f\left(z_{2}\right), f\left(z_{3}\right)\right] \\
f\left(z_{\alpha}\right) & =\ln z_{\alpha} \\
z_{\alpha} & =x_{1}+p_{\alpha} x_{2}
\end{aligned}
$$


and $\mathbf{A}$ and $\mathbf{B}$ are the $3 \times 3$ normalized complex matrices determined by the material elastic stiffness tensor, $\boldsymbol{\psi}$ is a stress function, and $p_{\alpha}$ are the eigenvalues with positive imaginary parts defined in the Stroh formalism assuming all the eigenvalues are distinct (for details, see Ref. [14, 19]). The explicit expression for the stress field is

$$
\begin{aligned}
& {\left[\sigma_{11}, \sigma_{21}, \sigma_{31}\right]^{\mathrm{T}}=-\boldsymbol{\psi}_{, 2}=-\frac{1}{\pi} \operatorname{Im}\left\{\mathbf{B} \mathbf{P} \boldsymbol{\Lambda} \mathbf{B}^{\mathrm{T}}\right\} \mathbf{b}} \\
& {\left[\sigma_{12}, \sigma_{22}, \sigma_{32}\right]^{\mathrm{T}}=\boldsymbol{\psi}_{, 1}=\frac{1}{\pi} \operatorname{Im}\left\{\mathbf{B} \boldsymbol{\Lambda} \mathbf{B}^{\mathrm{T}}\right\} \mathbf{b}}
\end{aligned}
$$

where

$$
\begin{aligned}
& \mathbf{P}=\operatorname{diag}\left[p_{1}, p_{2}, p_{3}\right] \\
& \boldsymbol{\Lambda}=\operatorname{diag}\left[1 / z_{1}, 1 / z_{2}, 1 / z_{3}\right]
\end{aligned}
$$

The elastic self-energy of a dislocation per unit length within a cylindrical region between $r_{0}$ and $R$ is found as the strain energy within the volume due to the dislocation elastic field, i.e.,

$$
E_{\mathrm{e}}=\frac{1}{2} \int_{V} \boldsymbol{\sigma}: \varepsilon \mathrm{d} V
$$

The volume integral of the strain energy density is equivalent to the work done by the traction over the surface enclosing the volume as

$$
E_{\mathrm{e}}=\frac{1}{2} \int_{\Omega} \mathbf{u}^{\mathrm{T}} \cdot \boldsymbol{\sigma} \cdot \mathbf{n} \mathrm{d} s
$$

where the integration is carried out along the path enclosing the volume $V$ and excluding any discontinuity in $\mathbf{u}$ due to the cut plane and singularity in $\sigma$ at the dislocation core, i.e. along the circular path around the core $\mathrm{S}^{(\mathrm{C})}$, the cut plane $\mathrm{S}^{( \pm)}$, and the outer radius $\mathrm{S}^{(\mathrm{R})}$ (see Fig. 1c). The total energy is independent of the choice of the cut plane. The path integral around $\mathrm{S}^{(\mathrm{C})}$ is the core traction contribution to the elastic energy. When the cylinder under consideration is embedded inside an infinite elastic domain, the path integral around $\mathrm{S}^{(\mathrm{C})}$ is exactly the opposite of the integral around $\mathrm{S}^{(\mathrm{R})}[12,13]$. Therefore, only the path integral along the cut plane $\mathrm{S}^{( \pm)}$contributes to the elastic self-energy and can be calculated as

$$
E_{\mathrm{e}}=\frac{1}{2} \int_{r_{0}}^{R} \sigma_{i 2}\left(x_{1}, 0\right) b_{i} \mathrm{~d} x_{1}=\frac{1}{2 \pi} \mathbf{b}^{\mathrm{T}} \operatorname{Im}\left\{\mathbf{B B}^{\mathrm{T}}\right\} \mathbf{b} \ln \frac{R}{r_{0}}=K \ln \frac{R}{r_{0}}
$$


where $K$ is the elastic self-energy prefactor completely determined by the Burgers vector and elastic stiffness tensor of the crystal. The elastic self-energy diverges as $R \rightarrow \infty$ due to the long-range $1 / r$ stress field and as $r_{0} \rightarrow 0$ due to the limitation of linear elasticity in modeling the core region where deformation is large and nonlinear, as is well known. The total dislocation energy can thus be written as

$$
E_{\mathrm{tot}}=E_{\mathrm{c}}\left(r_{0}\right)+K \ln \frac{R}{r_{0}}
$$

where $E_{\mathrm{c}}\left(r_{0}\right)$ corrects the core energy relative to the linear elastic energy using the core-cutoff $r_{0}$. All of the above is well-known, and repeated here only to establish notation and definitions needed for subsequent computations.

\subsection{Interaction energy of two straight dislocations}
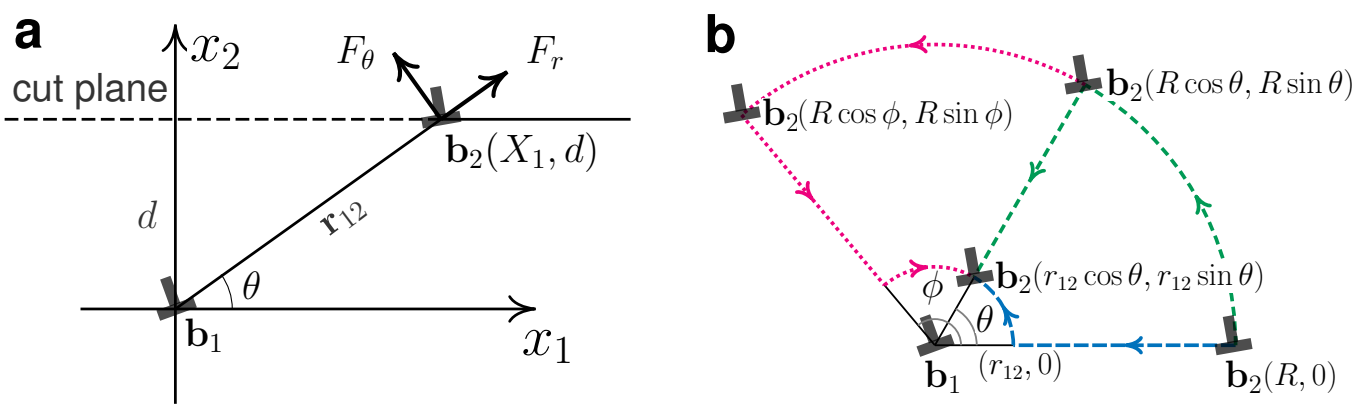

Figure 2: (a) Coordinate system for the interaction of two straight dislocations located at $(0,0)$ and $\left(X_{1}, d\right)$. (b) Path-independence of the interaction energy between the two dislocations. The interaction energy between $\mathbf{b}_{1}$ at the origin and $\mathbf{b}_{2}$ at $\left(r_{12} \cos \theta, r_{12} \sin \theta\right)$ can be calculated following different paths as indicated, (i) $(R, 0) \rightarrow\left(r_{12}, 0\right) \rightarrow\left(r_{12} \cos \theta, r_{12} \sin \theta\right)$, or (ii) $(R, 0) \rightarrow(R \cos \theta, R \cos \theta) \rightarrow\left(r_{12} \cos \theta, r_{12} \sin \theta\right)$ or $($ iii) $(R, 0) \rightarrow(R \cos \phi, R \cos \phi) \rightarrow$ $\left(r_{12} \cos \phi, r_{12} \sin \phi\right) \rightarrow\left(r_{12} \cos \theta, r_{12} \sin \theta\right)$, with the interaction energy independent of the path.

We now consider two dislocations having the same line direction $\boldsymbol{\xi}$ along the $x_{3}$ axis, with one dislocation of Burgers vector $\mathbf{b}_{1}$ located at the origin and the second dislocation $\mathbf{b}_{2}$ located at position $\left(r_{12}, \theta\right)$ or $\left(X_{1}, d\right)$, as shown in Fig. 2 a. The elastic interaction energy between two parallel straight dislocations can be calculated as the work done in moving (or creating) the second dislocation from infinity to its final position against the stress field generated by the first dislocation [20, 21, 13]. In practice, we must select an initial position for the second dislocation at polar coordinates $\left(R, \theta^{\prime}\right)$ from the origin, and compute the work 
done in moving the second dislocation to its final position. We will later consider the energy difference between two different final states, and this energy difference is independent of the choice of $\left(R, \theta^{\prime}\right)$ and so we choose $\theta^{\prime}=0$ for simplicity. The elastic interaction energy can thus be written as the integral of the work done [22] along a path $C$,

$$
W_{\mathrm{e} 12}=\int_{C}\left(\boldsymbol{\sigma}^{(1)} \cdot \mathbf{b}_{2}\right) \times \boldsymbol{\xi} \cdot \mathrm{d} \mathbf{s}, \mathrm{d} \mathbf{s}=\mathrm{d} \mathbf{r}+r \mathrm{~d} \boldsymbol{\theta}
$$

where $\boldsymbol{\sigma}^{(1)}$ is the stress tensor of dislocation $\mathbf{b}_{1}$ at the origin. Since the dislocation stress field (Eq.5) can always be written in the form

$$
\boldsymbol{\sigma}=\frac{1}{r} \mathbf{f}(\theta)
$$

the integral above can always be decomposed into two parts, a contribution $W_{\mathrm{e} 12}(R)$ due to the radial integral that scales as $\ln \left(R / r_{12}\right)$ and a contribution $W_{\mathrm{e} 12}(\theta)$ due to the angular integral that is independent of $R$ or $r_{12}$. This simple analysis unambiguously demonstrates the existence of the angular contribution. The interaction energy is independent of the path taken from the initial to final positions. Fig. 2b shows several paths following various combinations of radial and angular paths, and path-independence is clear when the interaction energy has the form $W_{\mathrm{e} 12}=W_{\mathrm{e} 12}(R)+W_{\mathrm{e} 12}(\theta)$.

For ease of computations given the final position $\left(X_{1}, d\right)$ (Fig. 2a), we choose a path with an angular part over angle $\Delta \theta=d / R$ at $R$, which is negligible as $R \rightarrow \infty$, followed by a straight path along $x_{2}=d$ from $x_{1}=\sqrt{R^{2}-d^{2}}$ to $x_{1}=X_{1}$. The work done is then

$$
W_{\mathrm{e} 12}=\int_{X_{1}}^{\sqrt{R^{2}-d^{2}}} b_{i}^{(2)} \sigma_{i 2}^{(1)}\left(x_{1}, d\right) \mathrm{d} x_{1}
$$

where $b_{i}^{(2)}$ is the component of the Burgers vector of dislocation $\mathbf{b}_{2}, \sigma_{i 2}^{(1)}$ is the stress component of dislocation $\mathrm{b}_{1}$ at the origin, as shown earlier in Eq. 5b. Carrying out the integration in Eq. 13 gives

$$
\begin{aligned}
W_{\mathrm{e} 12}= & \int_{X_{1}}^{\sqrt{R^{2}-d^{2}}} \mathbf{b}_{2}^{\mathrm{T}} \frac{1}{\pi} \operatorname{Im}\left\{\mathbf{B} \boldsymbol{\Lambda} \mathbf{B}^{\mathrm{T}}\right\} \mathbf{b}_{1} \mathrm{~d} x_{1}=\frac{1}{\pi} \mathbf{b}_{2}^{\mathrm{T}} \operatorname{Im}\left\{\mathbf{B} \mathbf{B}^{\mathrm{T}}\right\} \mathbf{b}_{1} \ln \frac{R}{r_{12}} \\
& -\frac{1}{\pi} \mathbf{b}_{2}^{\mathrm{T}} \operatorname{Im}\left\{\mathbf{B}\left\langle\ln \left(\cos \theta+p_{\alpha} \sin \theta\right)\right\rangle \mathbf{B}^{\mathrm{T}}\right\} \mathbf{b}_{1}=K_{12} \ln \frac{R}{r_{12}}-E_{\theta}
\end{aligned}
$$


As expected, the interaction energy has two additive terms representing the radial $\mathbf{r}$ and angular $\boldsymbol{\theta}$ contributions. This result was first obtained by Nabarro [21] in isotropic elasticity and by Stroh [13] in anisotropic elasticity. Also, the angular term is zero for the interaction between pure screw dislocation interactions in isotropic elasticity $[1,18]$. By using the very general Stroh formalism, both the $K_{12}$ coefficient of the radial term and the angular energy emerge very simply.

The interaction energy implies configurational interaction forces on the second dislocation (with the first dislocation held fixed). The configurational interaction forces in the radial and angular directions are

$$
\begin{aligned}
& f_{r}=-\frac{\mathrm{d} W_{12}}{\mathrm{~d} r_{12}}=\frac{K_{12}}{r_{12}} \\
& f_{\theta}=-\frac{\mathrm{d} W_{12}}{r_{12} \mathrm{~d} \theta}=\frac{1}{\pi r_{12}} \mathbf{b}_{2}^{\mathrm{T}} \operatorname{Im}\left\{\mathbf{B}\left\langle\frac{p_{\alpha} \cos \theta-\sin \theta}{\cos \theta+p_{\alpha} \sin \theta}\right\rangle \mathbf{B}^{\mathrm{T}}\right\} \mathbf{b}_{1}
\end{aligned}
$$

The above analysis applies to the interaction among partial dislocations $\left(\mathbf{b}_{1}, \mathbf{b}_{2}\right)$ and $\left(\mathbf{b}_{1}^{\prime}, \mathbf{b}_{2}^{\prime}\right)$ as the two dissociated partials. For glide dissociation, i.e. two partials on the same glide plane, a repulsive radial force acts along the glide plane and an equilibrium spacing is achieved when the radial force is balanced by the attractive configurational SF force. The angular force acts perpendicular to the glide plane and is counteracted by atomic bending distortion across the SF [23]. The angular force thus tends to drive cross-slip or climb of the partials, as well as bending of SF planes, all as seen in previous MD simulations [6].

\subsection{Energetics of dissociated dislocation transformations}

With the elastic self-energy and interaction energy determined in Eq. 9 and 14, the total energy of a pair of dislocations, using Eq. 1, can be approximated as

$$
\begin{aligned}
E_{\mathrm{tot}} & =\left[E_{\mathrm{c} 1}\left(r_{01}\right)+E_{\mathrm{c} 2}\left(r_{02}\right)\right]+\left[K_{1} \ln \frac{R}{r_{01}}+K_{2} \ln \frac{R}{r_{02}}+K_{12} \ln \frac{R}{r_{12}}-E_{\theta}\right]+\gamma_{\mathrm{sf}} r_{12} \\
& =\left[E_{\mathrm{c} 1}\left(r_{01}\right)+E_{\mathrm{c} 2}\left(r_{02}\right)\right]+\left[K \ln R-K\left(\ln r_{01}^{\bar{K}_{1}} r_{02}^{\bar{K}_{2}} r_{12}^{\bar{K}_{12}}\right)-E_{\theta}\right]+\gamma_{\mathrm{sf}} r_{12}
\end{aligned}
$$

Here, the three groups of terms are the core energy, the elastic energy, and the stacking fault energy. In the elastic energy, $K, K_{1}, K_{2}$ and $K_{12}$ are the energy prefactors for the total dislocation, the individual partial dislocations, and their elastic interaction, and $\bar{K}_{\alpha}=K_{\alpha} / K$ is a normalized prefactor. The sum rule $K=K_{1}+K_{2}+K_{12}$ holds for any dissociation, since $K$ is completely determined by the topological character of the dislocation $(\mathbf{b}, \boldsymbol{\xi})$ and elastic properties of the 
materials $\left(C_{i j}\right)$, independent of the dissociation structure. Also, $r_{01}, r_{02}$ are the two partial dislocation core radii and $r_{12}$ is the partial separation. The $K \ln R$ term in Eq. 16 captures the far field elastic energy of the entire dislocation. Therefore, the total energy can only be reduced through structure transformations achieved by changes in the remaining terms associated with changes in the partial Burgers vectors, orientation, and SF energy. Of course, the SF energy $\gamma_{\mathrm{sf}}$ is determined by the plane defined by $\theta$ and viable choices for $\theta$ are limited to planes that have a metastable SF. For glide dissociations, the equilibrium dissociation distance is $r_{12}=K_{12} / \gamma_{\text {sf }}$ and so the SF contribution to the total energy at equilibrium is precisely $K_{12}$, completely determined by the Burgers vectors of the partials and elastic constants, and independent of the SF energy. However, lower SF energies result in wider partial separations, which reduce the partial interaction energy and thus the total dislocation energy. For climb dissociation, $r_{12}$ should be limited by kinetics during the climb process, and is not directly related to the SF energy. Overall, core structure transitions are driven by reduction in near-core dislocation energy under the constraints set by crystal structure and climb kinetics.

If we assume $r_{01} \approx r_{02}=r_{0}$ for cases where $\mathbf{b}_{1} \approx \mathbf{b}_{2}$, Eq. 16 further reduces to

$$
E_{\mathrm{tot}}=\left[E_{\mathrm{c} 1}\left(r_{0}\right)+E_{\mathrm{c} 2}\left(r_{0}\right)\right]+\left[K \ln \frac{R}{r_{0}}-K_{12} \ln \frac{r_{12}}{r_{0}}-E_{\theta}\right]+\gamma_{\mathrm{sf}} r_{12}
$$

where $K_{12} \ln \left(r_{12} / r_{0}\right)$ is the change of the radial part of the interaction energy, but not the interaction energy itself.

For two different dissociations of the same dislocation, as shown in Fig. 1a and $b$, the energy difference between them, using Eq. 16, can now be written as

$$
\begin{aligned}
\Delta E_{\mathrm{tot}}= & {\left[E_{\mathrm{c} 1}^{\prime}\left(r_{01}^{\prime}\right)+E_{\mathrm{c} 2}^{\prime}\left(r_{02}^{\prime}\right)-E_{\mathrm{c} 1}\left(r_{01}\right)-E_{\mathrm{c} 2}\left(r_{02}\right)\right] } \\
& +\left[K \ln \left(r_{01}^{\bar{K}_{1}} r_{02}^{\bar{K}_{2}} r_{12}^{\bar{K}_{12}}\right)-K \ln \left(r_{01}^{\prime \bar{K}^{\prime}{ }_{1}} r_{02}^{\prime} \bar{K}^{\prime_{2}}{ }_{12}^{\prime}{ }^{\bar{K}^{\prime}{ }_{12}}\right)\right] \\
& +\left[E_{\theta}-E_{\theta^{\prime}}^{\prime}\right]+\left[\gamma_{\mathrm{sf}}^{\prime} r_{12}^{\prime}-\gamma_{\mathrm{sf}} r_{12}\right] \\
= & \Delta E_{\mathrm{c}}+\Delta E_{\mathrm{r}}+\Delta E_{\theta}+\Delta E_{\mathrm{sf}}
\end{aligned}
$$

where the unprimed and primed symbols denote quantities corresponding to the first and second dissociation configurations, respectively. In Eq. 18, the energy terms are grouped by their physical origins. The first group represent the differences in core energies of the partials, which are dictated by the detailed structures of the partial core; these can not be determined by elastic theory. For the cases considered here, we consider transformations where $\mathbf{b}_{1} \approx \mathbf{b}_{1}^{\prime}$ and $\mathbf{b}_{2} \approx \mathbf{b}_{2}^{\prime}$ and 
assume this term to be small, i.e. the core energies of the partial dislocations do not change significantly upon transformation. We validate this assumption for several cases in $\mathrm{Mg}$ by comparing the elastic predictions to direct atomistic values measured in previous $\mathrm{Mg}$ simulations [6,7]. The second group of terms involving the $K \ln r_{0 i}^{\bar{K}_{i}}$ represent the elasticity cut-offs that are corrected by the core energy, and this term is generally small if the core cut-offs scale with the Burgers vectors of the various partials. However, the term $K \ln r_{12}^{\bar{K}_{12}}$ captures the partial elastic interaction at distance $r_{12}$, which can vary substantially due to large variations in $r_{12}$ (due to $\gamma_{\mathrm{sf}}$ or climb kinetics) among different dissociations. The third and fourth groups in $\Delta E_{\text {tot }}$ represent the changes in the angular interaction energy and the SF energy, respectively. All the terms except the core energy in Eq. 18 can be evaluated quantitatively in terms of the crystal elastic constants, partial dislocation Burgers vectors, and SF energy. As required physically, the change in total energy due to different dissociations is independent of the far field elastic energy and thus independent of $R$.

The above formulation contained in Eq. 16 and 18 completely ignores any core effects and should be generally accurate in comparing dislocation dissociations where the changes in Burgers vectors are small. In addition, all terms can be computed analytically given the solution of the eigenvalue problem in the Stroh formulation of anisotropic elasticity. However, the elastic self-energy in Eq. 9 is only valid for a single dislocation in an infinite domain, i.e. when the core traction contribution is cancelled by the traction contribution at the outer radius $R$. For a pair of arbitrary dislocations, the individual core traction contributions generally do not cancel with the traction contribution in the far field. This makes the dislocation total energy calculated in Eq. 16 weakly dependent on the cut plane chosen in the formulation. A cut-plane-independent solution is obtained only when the tractions at the dislocation core and the far field are explicitly taken into account $[24,18]$. We show the exact cut-plane-independent formulation for a general dislocation pair in Appendix. With that, the total elastic energy of a pair of arbitrary dislocations consists of the elastic self energy of the individual dislocations in infinite domain, their elastic interactions, and the traction contributions from the individual cores and from the far field. The total energy per unit length 
of any dissociated dislocation can thus be written, using Eq. 1, as

$$
\begin{aligned}
E_{\mathrm{tot}}= & {\left[E_{\mathrm{c} 1}\left(r_{01}\right)+E_{\mathrm{c} 2}\left(r_{02}\right)\right] } \\
& +\left[K_{1} \ln \frac{R}{r_{01}}+K_{2} \ln \frac{R}{r_{02}}+K_{12} \ln \frac{R}{r_{12}^{*}}-E_{\theta^{*}}+E_{\mathrm{e}}^{\mathrm{S}^{(\mathrm{C} 1)}}+E_{\mathrm{e}}^{\mathrm{S}^{(\mathrm{C} 2)}}+E_{\mathrm{e}}^{\mathrm{S}^{(\mathrm{R})}}\right]+\gamma_{\mathrm{sf}} r_{12} \\
= & {\left[E_{\mathrm{c} 1}+E_{\mathrm{c} 2}\right]+\left[K \ln R-K\left(\ln r_{01}^{\bar{K}_{1}} r_{02}^{\bar{K}_{2}} r_{12}^{* \bar{K}_{12}}\right)-E_{\theta^{*}}+E_{\mathrm{e}}^{\mathrm{S}^{(\mathrm{C} 1)}}+E_{\mathrm{e}}^{\mathrm{S}^{(\mathrm{C} 2)}}+E_{\mathrm{e}}^{\mathrm{S}^{(\mathrm{R})}}\right]+\gamma_{\mathrm{sf}} r_{12} }
\end{aligned}
$$

Here, the three groups of terms are the core energy, the elastic energy, and the stacking fault energy, same as that in Eq. 16. In the elastic energy, $E_{\mathrm{e}}^{\mathrm{S}^{(\mathrm{C} 1)}}, E_{\mathrm{e}}^{\mathrm{S}^{(\mathrm{C} 2)}}$ and $E_{\mathrm{e}}^{\mathrm{S}^{(\mathrm{R})}}$ are the traction contributions at the two partial cores and far field, respectively. Also, $r_{12}^{*}$ and $\theta^{*}$ are defined in Fig. A.1b and Eq. A.8, and approach $r_{12}$ and $\theta$ when $r_{12}$ is large compared to the core radii $r_{0 i}$.

The energy difference between two dissociations, as shown in Fig. 1a and b, can then be written as

$$
\begin{aligned}
& \Delta E_{\mathrm{tot}}=\left[E_{\mathrm{c} 1}^{\prime}\left(r_{01}^{\prime}\right)+E_{\mathrm{c} 2}^{\prime}\left(r_{02}^{\prime}\right)-E_{\mathrm{c} 1}\left(r_{01}\right)-E_{\mathrm{c} 2}\left(r_{02}\right)\right] \\
& +\left[K \ln \left(r_{01}^{\bar{K}_{1}} r_{02}^{\bar{K}_{2}} r_{12}^{* \bar{K}_{12}}\right)-K \ln \left(r_{01}^{\prime}{ }^{\bar{K}^{\prime}}{ }_{1} r_{02}^{\prime}{ }^{\bar{K}^{\prime}}{ }_{2} r_{12}^{* \prime} \bar{K}^{\prime}{ }_{12}\right)\right] \\
& +\left[E_{\theta^{*}}-E_{\theta^{*^{\prime}}}^{\prime}\right]+\left[\gamma_{\mathrm{sf}}^{\prime} r_{12}^{\prime}-\gamma_{\mathrm{sf}} r_{12}\right] \\
& +\left[E_{\mathrm{e}}^{\prime \mathrm{S}^{(\mathrm{C} 1)}}+E_{\mathrm{e}}^{\prime \mathrm{S}^{(\mathrm{C} 2)}}-E_{\mathrm{e}}^{\mathrm{S}^{(\mathrm{C} 1)}}-E_{\mathrm{e}}^{\mathrm{S}^{(\mathrm{C} 2)}}\right] \\
& =\Delta E_{\mathrm{c}}+\Delta E_{\mathrm{r}}+\Delta E_{\theta}+\Delta E_{\mathrm{sf}}+\Delta E_{\mathrm{ct}}
\end{aligned}
$$

where the unprimed and primed symbols denote quantities corresponding to the first and second dissociation configurations, respectively. The first four terms have similar physical origins as those in Eq. 18 (see Appendix for details). The last group are the elastic energy differences due to the traction contributions at the dislocation cores. The traction integral at the outer radius $R \gg r_{12}$ is equivalent to that of the undissociated single dislocation and thus is the same for the two dissociations. All the terms except the core energy in Eq. 20 can be evaluated quantitatively in terms of the crystal elastic constants, partial dislocation Burgers vectors, and SF energy.

We have now presented two approaches, Eq. 18 and 20, for comparing the total energy differences. The first approach is analytical and physically clear, but depends weakly on the cut plane. The second approach, using the general surface traction integral, is exact and cut-plane-independent, but is far more difficult to compute. Nevertheless, both approaches should yield similar results, particularly when the changes in the Burgers vectors are small and the partial separation $r_{12}$ is 
large compared to the core radii $r_{0 i}$, i.e. when the elastic analysis is appropriate (we advocate use of the first analytical approach in such cases). Below, we show quantitative comparisons of the two approaches and show the differences to be small (i.e. at a level where neglect of core energies is a more serious limitation of the analysis).

\section{Transformations of dissociated dislocations in hep metals}

We now analyze dislocation dissociations for $6 \mathrm{hcp}$ metals $(\mathrm{Mg}, \mathrm{Ti}, \mathrm{Zr}, \mathrm{Re}, \mathrm{Zn}$ and Cd) spanning 4 columns in the periodic table. The analysis is presented in detail for four dissociations relevant to current experimental observations and atomistic simulations, and for which the Burgers vector changes upon transformation are small so that our analysis is appropriate. The first two cases, shown in Fig.4a and Fig.5a, compare the energies of the mixed and edge $\langle\mathbf{c}+\mathbf{a}\rangle$ dislocations when dissociated on Pyr. I and II plane, respectively, versus the climb-dissociated structures oriented on the Basal planes. Recent MD simulations [6, 7] and isotropic elasticity analysis [25] (edge $\langle\mathbf{c}+\mathbf{a}\rangle$ ) show that $\langle\mathbf{c}+\mathbf{a}\rangle$ climb-dissociations on Basal planes are energetically favorable for $\mathrm{Mg}$, and that the energy barrier is low $[6,7]$. The third case (Fig. 6a) compares the energies of the screw $\langle\mathbf{c}+\mathbf{a}\rangle$ dissociated on Pyr. I and II planes, where all partials retain similar near-pure-screw character. The last case (Fig. 7a) examines the screw $\langle\mathbf{a}\rangle$ dislocation dissociations on the Basal, Prism and Pyr. I planes, where again the partials retain similar near-pure-screw character on the Prism and Pyr. I planes. Comparing the energies of screw $\langle\mathbf{a}\rangle$ and $\langle\mathbf{c}+\mathbf{a}\rangle$ dislocation dissociations helps determine their primary slip systems and ease of cross-slip, particularly in $\mathrm{Mg}$ and $\mathrm{Ti}$ where multiple slip planes may operate and for which controversies on the importance of each system exist [26, 27, 28, 29, 10, 30, 15]. In addition, we also provide a preliminary analysis on $\langle\mathbf{c}+\mathbf{a}\rangle$ dissociating into $\langle\mathbf{c}\rangle$ and $\langle\mathbf{a}\rangle$ in separation, as shown in Fig. 8a and b.

As input to the calculations, we use the experimentally-measured lattice and elastic constants $[32,31]$ shown in Table 1 . The elastic constants were measured at $\sim 4 \mathrm{~K}$ [32]. The lattice constant $a$ is calculated based on material density at $\sim 4 \mathrm{~K}$ [32] and room temperature $c / a$ ratio [31]. We also use the relevant SF energies $\left(\gamma_{\mathrm{sf}}\right)$ and positions of the relevant stable SF for the 6 hcp metals as computed by carefully converged DFT calculations using the Vienna Ab initio Simulation Package (VASP) [34, 35]. The DFT calculations employ a plane-wave spin free basis set in the generalized gradient approximation (GGA) parametrized by Perdew-Burke-Ernzerhof (PBE) [36] and with core electrons replaced by projec- 


\begin{tabular}{llllllll}
\hline & $\mathbf{M g}(\mathbf{M E A M})$ & $\mathbf{M g}$ & $\mathbf{T i}$ & $\mathbf{Z r}$ & $\mathbf{R e}$ & $\mathbf{Z n}$ & $\mathbf{C d}$ \\
\hline & & & & & & & \\
$a$ & 3.187 & 3.186 & 2.947 & 3.228 & 2.760 & 2.647 & 2.957 \\
$c / a$ & 1.623 & 1.624 & 1.586 & 1.593 & 1.615 & 1.856 & 1.886 \\
& & & & & & & \\
$C_{11}$ & 64.27 & 63.48 & 176.1 & 155.4 & 634.4 & 179.1 & 129.2 \\
$C_{12}$ & 25.45 & 25.94 & 86.90 & 67.20 & 266.0 & 37.50 & 39.99 \\
$C_{13}$ & 20.29 & 21.70 & 68.30 & 64.60 & 202.0 & 55.40 & 40.95 \\
$C_{33}$ & 70.93 & 66.45 & 190.5 & 172.5 & 701.6 & 68.80 & 56.68 \\
$C_{44}$ & 18.02 & 18.42 & 50.80 & 36.30 & 169.1 & 45.95 & 24.20 \\
\hline
\end{tabular}

Table 1: Lattice and elastic constants of hep metals. Values of lattice parameter $a(\AA)$ are scaled to $4 \mathrm{~K}$ with $c / a$ fixed [31, 32]. Elastic constants $C_{i j}$ (GPa) are material properties at $4 \mathrm{~K}$ [32]. The elastic constants are given with $x_{3}$-axis in the c-axis. Mg (MEAM) are properties of the MEAM potential [33] at $0 \mathrm{~K}$.
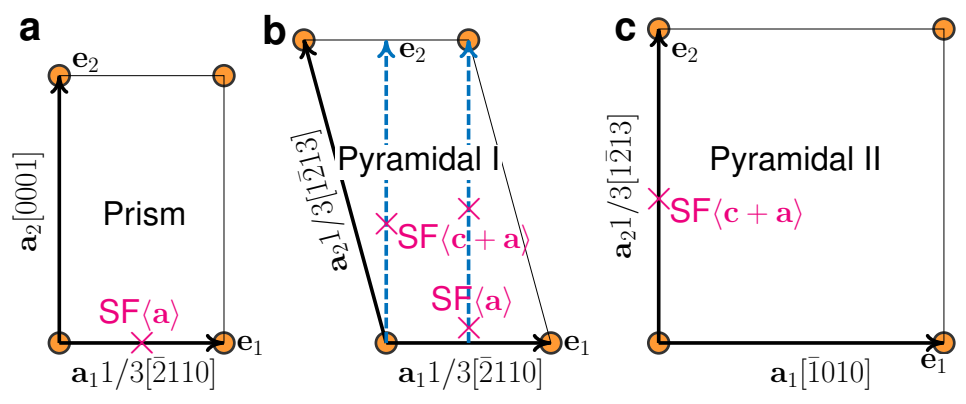

Figure 3: Schematics of stable stacking fault positions on (a) Prism, (b) Pyramidal I and (c) Pyramidal II planes. $\mathbf{e}_{1}$ and $\mathbf{e}_{2}$ are the unit vectors of the Cartesian coordinate system, while $\mathbf{a}_{1}$ and $\mathbf{a}_{2}$ are the lattice vectors of the simulation unit cell. 
tor augmented wave (PAW) pseudopotentials [37] with the valence states shown in Table 2. Tilted boxes with periodic boundary conditions and full atom relaxation $[38,39,40]$ are used to determine the SF energies and positions. Further details, more complete results, and extensive discussion regarding the SF structures are presented elsewhere. However, for clarity, Figure 3 and Table 2 show the calculated SF positions and energies; for planes with multiple stacking faults, only those with the lowest SF energies are shown because they are relevant for the current analysis. The DFT calculations show that the metastable SF relevant to $\langle$ a $\rangle$ dislocations on the Prism plane only exists in $\mathrm{Ti}, \mathrm{Zr}$ and Re. Therefore, in $\mathrm{Mg}$, $\mathrm{Zn}$ and $\mathrm{Cd}$, we take the unstable SF energy at $(\mathrm{a} / 2,0)$ as a hypothetical metastable $\mathrm{SF}$, imagining the possibility of a very shallow minimum not resolved by DFT (see Ref. [41]). To compare the analysis with direct atomistic simulations, properties of an MEAM potential for $\mathrm{Mg}$ [33] at $\mathrm{T}=0 \mathrm{~K}$ are also used, as shown. Unless otherwise stated, the elastic cutoff $r_{0}$ for all partials is taken as $|\mathbf{a}| / 2$ and $|\mathbf{c}+\mathbf{a}| / 2$ for $\langle\mathbf{a}\rangle$ and $\langle\mathbf{c}+\mathbf{a}\rangle$ dislocations, respectively. The equilibrium partial separations determined by elasticity, as shown in Table 3, are used in all calculations involving glide dissociations. For the two climb-dissociation cases, dislocation dissociation on the Basal plane requires self-compensated climb between the partials which is kinetically difficult and so we analyze energy differences as a function of the climb separation.

\begin{tabular}{|c|c|c|c|c|c|c|c|c|c|}
\hline & & & Mg(MEAM) & $\mathbf{M g}$ & $\mathbf{T i}$ & $\mathbf{Z r}$ & $\mathbf{R e}$ & $\mathbf{Z n}$ & Cd \\
\hline valence states & & & & $3 \mathrm{~s}$ & $4 s, 3 d$ & $5 s, 4 d$ & $6 \mathrm{~s}, 5 \mathrm{~d}$ & $4 s, 3 d$ & $5 s, 4 d$ \\
\hline \multirow{2}{*}{ Basal } & $\mathrm{I}_{1}$ & $\gamma_{\mathrm{sf}}$ & 11 & 18 & 172 & 149 & 54 & 48 & 17 \\
\hline & $\mathrm{I}_{2}$ & $\gamma_{\mathrm{sf}}$ & 23 & 34 & 306 & 224 & 206 & 110 & 29 \\
\hline \multirow{2}{*}{ Prism } & \multirow{2}{*}{$\mathrm{SF}\langle\mathbf{a}\rangle$} & $\mathbf{b}_{\mathrm{p}}$ & $(0.5,0)$ & $(0.5,0) *$ & $(0.5,0)$ & $(0.5,0)$ & $(0.5,0)$ & $(0.5,0)^{*}$ & $(0.5,0)^{*}$ \\
\hline & & $\gamma_{\mathrm{sf}}$ & 219 & $212^{*}$ & 214 & 182 & 933 & $502 *$ & $238^{*}$ \\
\hline \multirow{3}{*}{ Pyr. I } & \multirow{2}{*}{$\mathrm{SF}\langle\mathbf{a}\rangle$} & $\mathbf{b}_{\mathrm{p}}$ & $(0.5,0.116)$ & $(0.5,0.116)$ & $(0.5,0.089)$ & $(0.5,0.105)$ & $(0.5,0.112)$ & $(0.5,-0.025)$ & $(0.489,-0.034)$ \\
\hline & & $\gamma_{\mathrm{sf}}$ & 171 & 165 & 197 & 158 & 653 & 328 & 171 \\
\hline & $\mathrm{SF}\langle\mathbf{c}+\mathbf{a}\rangle$ & $\mathbf{b}_{\mathrm{p}}$ & $(0,0.422)$ & $(0,0.403)$ & $(0,0.435)$ & $(0,0.431)$ & $(0,0.421)$ & $(0.5,0.487)$ & $(0.5,0.499)$ \\
\hline \multirow{2}{*}{ Pyr. II } & \multirow{2}{*}{$\mathrm{SF}\langle\mathbf{c}+\mathbf{a}\rangle$} & $\mathbf{b}_{\mathrm{p}}$ & $(0,0.479)$ & $(0,0.480)$ & $(0,0.446)$ & $(0,0.455)$ & $(0,0.434)$ & $(0,0.535)$ & $(0,0.541)$ \\
\hline & & $\gamma_{\mathrm{sf}}$ & 200 & 165 & 321 & 282 & 1022 & 119 & 47 \\
\hline
\end{tabular}

Table 2: Relevant stacking fault energies and positions as calculated by DFT for various hcp metals and by an MEAM potential for Mg. The stacking fault energy and position, $\gamma_{\mathrm{sf}}$ and $\mathbf{b}_{\mathrm{p}}$, are in the units of $\mathrm{mJ} / \mathrm{m}^{2}$ and $\left(\mathbf{a}_{1} \cdot \mathbf{e}_{1}, \mathbf{a}_{2} \cdot \mathbf{e}_{2}\right.$, see Fig. 3), respectively. On the Prism plane, the stacking faults denoted by ' $*$ ' are unstable stacking faults in the DFT calculations, taken as proxies for possible shallow minima that are not found in the DFT calculations. 


\begin{tabular}{lllllllll}
\hline & & $\mathbf{M g}(\mathbf{M E A M})$ & $\mathbf{M g}$ & $\mathbf{T i}$ & $\mathbf{Z r}$ & $\mathbf{R e}$ & $\mathbf{Z n}$ & $\mathbf{C d}$ \\
\hline \multirow{2}{*}{$\operatorname{mixed}\langle\mathbf{c}+\mathbf{a}\rangle$} & Pyr. I & 7.0 & 7.0 & 21.6 & 25.5 & 13.7 & 11.3 & 28.3 \\
edge $\langle\mathbf{c}+\mathbf{a}\rangle$ & Pyr. II & 6.5 & 7.6 & 9.7 & 10.0 & 10.5 & 17.4 & 35.1 \\
& & & & & & & & \\
Screw $\langle\mathbf{c}+\mathbf{a}\rangle$ & Pyr. I & 4.9 & 5.0 & 14.6 & 16.9 & 9.6 & 10.5 & 23.0 \\
& Pyr. II & 4.7 & 5.5 & 6.6 & 6.8 & 7.4 & 16.8 & 29.8 \\
& & & & & & & & \\
Screw $\langle\mathbf{a}\rangle$ & Basal & 5.4 & 3.5 & 0.88 & 1.1 & 4.7 & 3.5 & 7.6 \\
& Prism & 1.1 & 1.1 & 2.6 & 2.8 & 2.1 & 1.2 & 1.6 \\
& Pyr. I & 1.0 & 1.0 & 2.4 & 2.5 & 2.2 & 1.8 & 2.2 \\
\hline
\end{tabular}

Table 3: Equilibrium glide dissociation distance calculated using lattice and elastic constants from experiments (Table. 1) and stacking fault energies from DFT (Table. 2). The distance is in the unit of the individual lattice parameter $a$. Mg (MEAM) is calculated using the properties of the MEAM potential [33] at $0 \mathrm{~K}$.

\subsection{Pyramidal-Basal $\langle\mathbf{c}+\mathbf{a}\rangle$ transformations}

Figure $4 \mathrm{~b}$ shows the energy difference between the Pyr. I dissociated mixed $\langle\mathbf{c}+\mathbf{a}\rangle$ and its Basal-dissociated counterpart, as a function of the Basal dissociation distance. Figure $5 b$ shows the energy difference between the Pyr. II dissociated edge $\langle\mathbf{c}+\mathbf{a}\rangle$ and its Basal-dissociated counterpart, as a function of the Basal dissociation distance. In all cases, the approximate and exact calculations are almost indistinguishable. For all six metals, dissociation on to the Basal plane is energetically favorable at accessible nanoscale climb distances. Further climb can then lead to large reductions in the Basal-dissociated dislocation energy. The critical climb distances are within $\sim 2 \mathrm{~nm}$ and $\sim 3 \mathrm{~nm}$ for both the mixed and edge $\langle\mathbf{c}+\mathbf{a}\rangle$ dislocations, respectively. These distances are well below their respective equilibrium distances, as evident from the slopes of the energy curves. For $\mathrm{Mg}$ (MEAM), the energy reductions based on elastic calculations at the measured climb distances $(\sim 2.5 \mathrm{~nm}$ for both Pyr. I and II) show remarkable agreement with the atomistically-measured values $[6,7]$. The direct simulations reinforce that nanoscale climb is feasible given a sufficiently large energetic driving force. The agreement of the elasticity analysis with the atomistically-measured MEAM results further indicates that the core energy differences among the different dissociations are small, demonstrating the robustness of the elastic analysis. The large energy reductions upon Basal climb dissociation show that the climb process is very likely to occur in all these metals. However, the rate of the transition will depend on the activation barrier for the process, and the mechanism of transition 

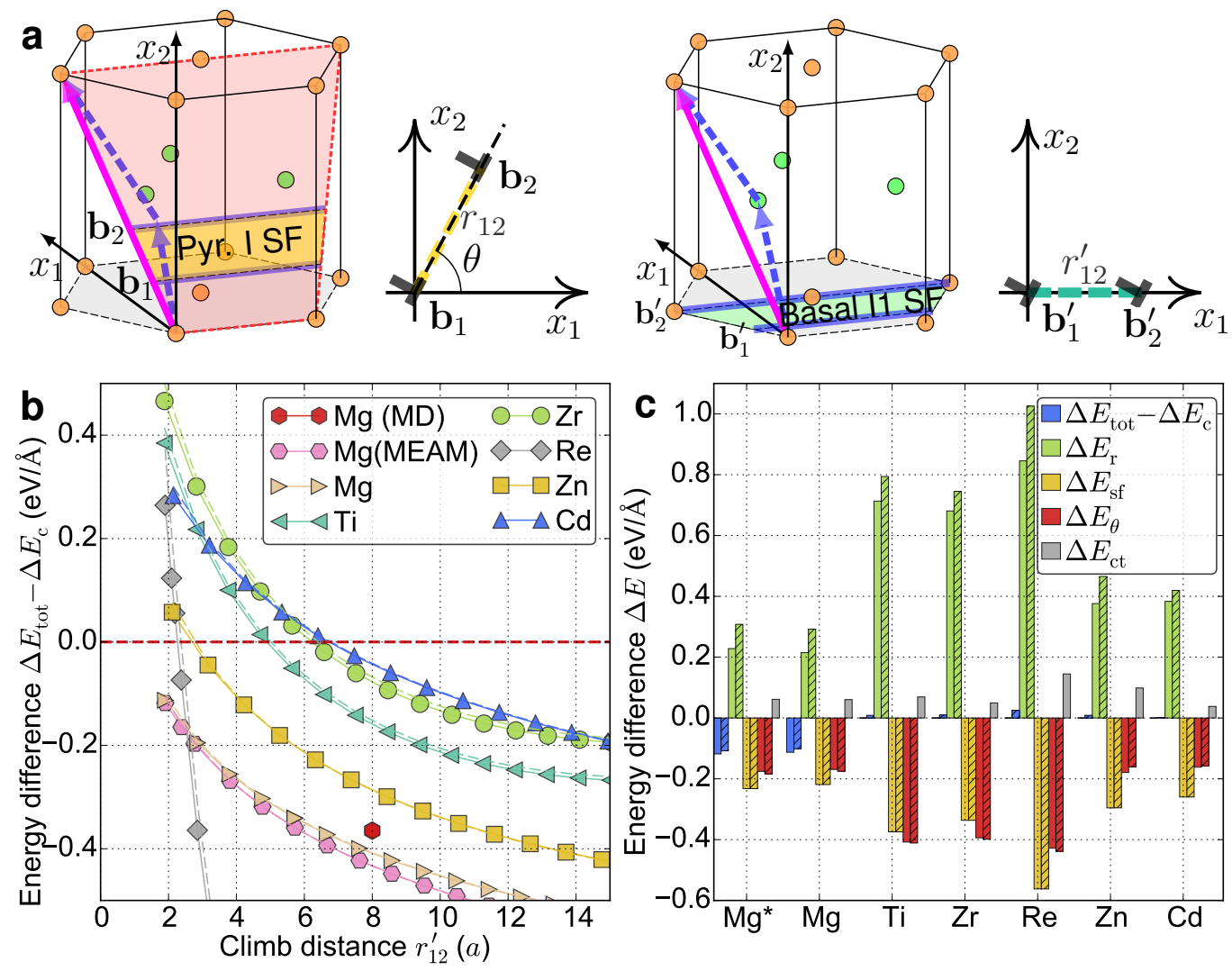

Figure 4: (a) Schematics and coordinate systems for mixed $\langle\mathbf{c}+\mathbf{a}\rangle$ dislocations dissociated on Pyramidal I and Basal planes. (b) Energy difference (Basal minus Pyramidal) as a function of climb distance. Basal dissociation is energetically favorable when the energy difference becomes negative. The results based on the surface traction integral and the approximations, denoted by the solid and dashed lines, respectively, are nearly identical. (c) Contributions to the total energy difference at the critical climb distance for all metals (except $\mathrm{Mg}$ and $\mathrm{Mg}^{*}$ (MEAM)) where the total energy difference is zero. The results of $\mathrm{Mg}$ and $\mathrm{Mg}^{*}$ is plotted at climb distance of $\mid \mathbf{c}+$ a|. The hatched bars show the approximate results using the critical climb distance of the exact calculation or $|\mathbf{c}+\mathbf{a}|$. The total energy differences of the two approaches (the blue bars) are nearly identical. The energy of Re is scaled by $1 / 3$. 
in hcp metals other than $\mathrm{Mg}$ is currently not entirely clear.
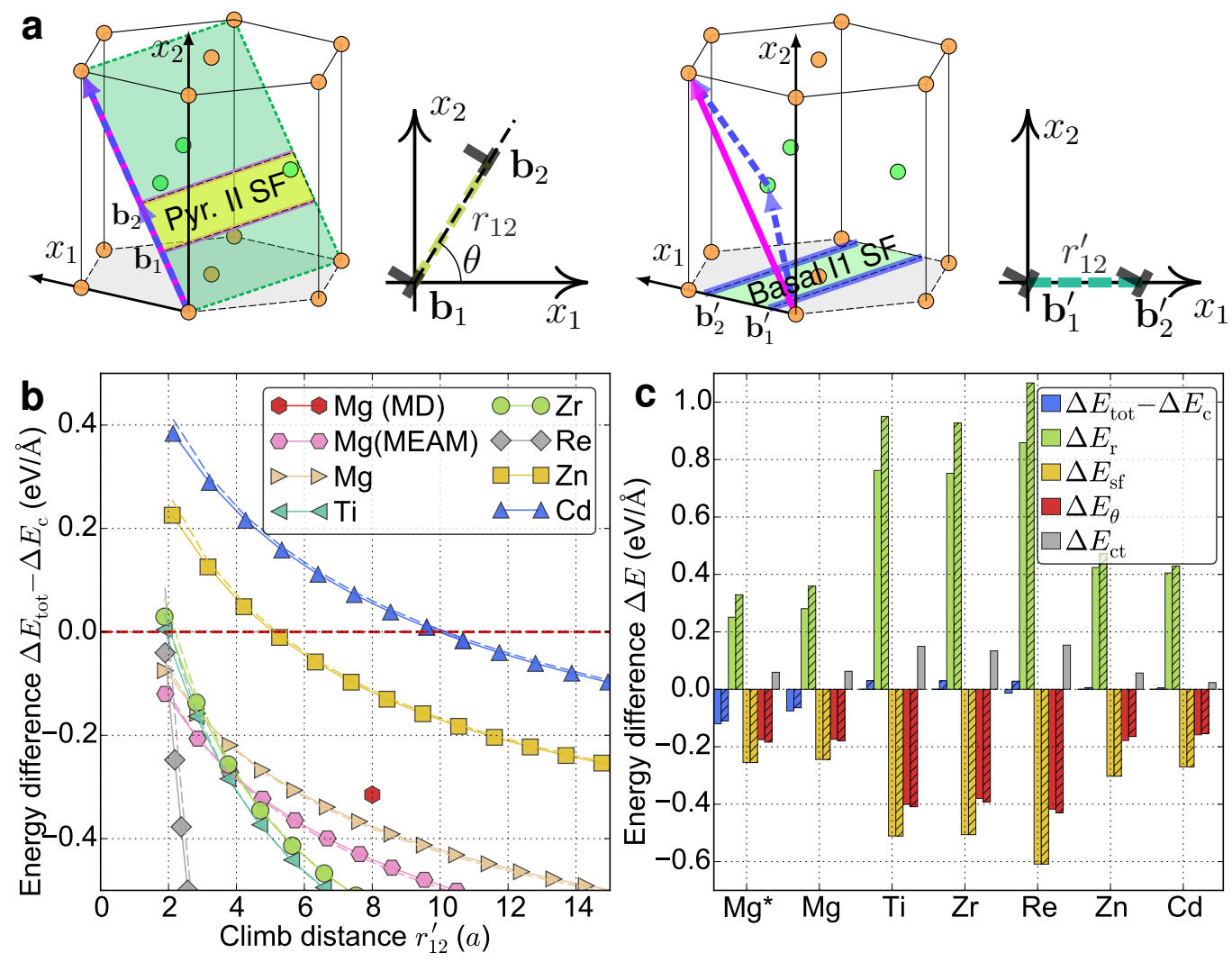

Figure 5: (a) Schematics and coordinate systems for edge $\langle\mathbf{c}+\mathbf{a}\rangle$ dislocations dissociated on Pyramidal II and Basal planes. (b) Energy difference (Basal minus Pyramidal) as a function of climb distance. Basal dissociation is energetically favorable when the energy difference becomes negative. The results based on the surface traction integral and the approximations, denoted by the solid and dashed lines, respectively, are nearly identical. (c) Contributions to the total energy difference at the critical climb distance for all metals (except Mg, Mg* (MEAM) and Re) where the total energy difference is zero. The results of $\mathrm{Mg}, \mathrm{Mg}^{*}$ and $\mathrm{Re}$ is plotted at climb distance of $|\mathbf{c}+\mathbf{a}|$. The hatched bars show the approximate results using the critical climb distance of the exact calculation or $|\mathbf{c}+\mathbf{a}|$. The total energy differences of the two approaches (the blue bars) are nearly identical. The energy of Re is scaled by $1 / 3$.

Figures $4 c$ and $5 c$ show the individual energy contributions to the total energy difference in the mixed and edge $\langle\mathbf{c}+\mathbf{a}\rangle$ cases, respectively, at the critical climb distance where the energy difference is zero or at a distance of $|\mathbf{c}+\mathbf{a}|$, noting that the angular term $E_{\theta}$ is independent of $r_{12}$. For all metals and both Pyr. I and II, 
the total energy differences using the approximate and exact elastic analyses are nearly identical. The angular term $E_{\theta}$ (red bar in Fig. $4 \mathrm{c}$ and $5 \mathrm{c}$ ) provides a large energy reduction $(\sim 0.15-1.31 \mathrm{eV} / \AA)$ upon climb-dissociation to the Basal plane. This is not surprising since the partial Burgers vectors consist of mainly edge components of the same sign, which strongly prefer a $90^{\circ}$ relative orientation, as suggested by isotropic dislocation elasticity. The energy contributions due to the change in SF energy and radial elastic interaction are coupled and depend on the selected Basal dissociation distance. For the chosen nanoscale Basal separations, well below the elastic equilibrium separations, the Basal-dissociation reduces the total SF energy but increases the radial elastic interaction energy. In the exact calculation, the energy differences due to the core traction integral are relatively small. In fact, the dominant term is contained in the radial part of the partial interaction energy in the approximate solution (see Appendix).

\subsection{Pyramidal I - II screw $\langle\mathbf{c}+\mathbf{a}\rangle$ transformations}
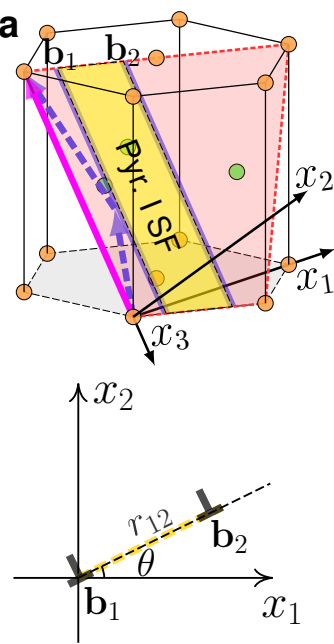
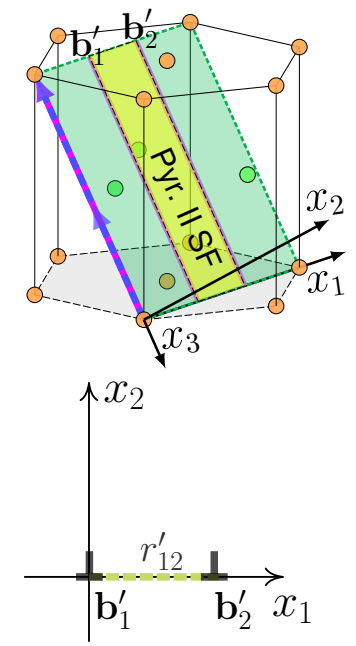

b

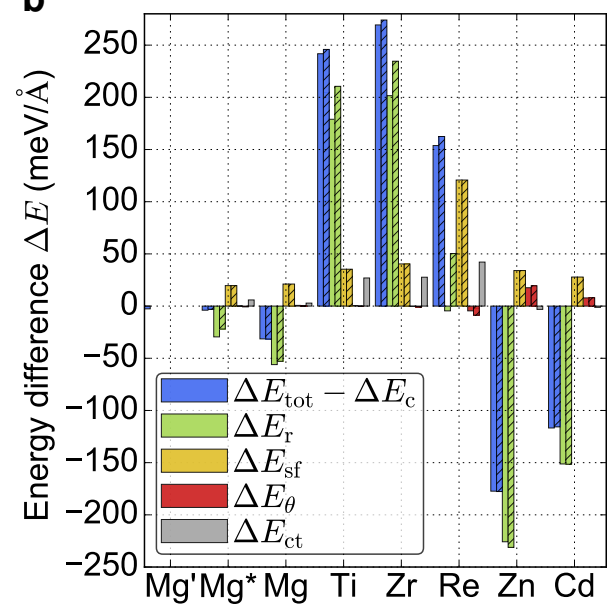

Figure 6: (a) Schematics and coordinate systems for screw $\langle\mathbf{c}+\mathbf{a}\rangle$ dislocations dissociated on Pyramidal I and II planes. (b) Total energy difference (Pyr. II - Pyr. I) and contributing components. Pyramidal II dissociation is energetically favorable when $\Delta E_{\text {tot }}-\Delta E_{\mathrm{c}}$ (the blue bar) is negative. $\mathrm{Mg}^{\prime}$ and $\mathrm{Mg}^{*}$ are the direct atomistic measurement and the elastic predictions using the MEAM potential [33], respectively. The hatched bars show the approximate results.

Figure $6 \mathrm{~b}$ shows the total energy difference and the associated contributing energies between a screw $\langle\mathbf{c}+\mathbf{a}\rangle$ dislocation dissociated on the Pyr. I and Pyr. II 
planes. In $\mathrm{Ti}, \mathrm{Zr}$ and $\mathrm{Re},\langle\mathbf{c}+\mathbf{a}\rangle$ dissociated on the Pyr. I plane has a substantially lower energy $(\sim 0.15-0.25 \mathrm{eV} / \AA)$ than that on Pyr. II planes, indicating that the Pyr. I plane is the primary $\langle\mathbf{c}+\mathbf{a}\rangle$ slip plane in these metals. This is consistent with experimental observations in $\mathrm{Ti}[42,8,43,44,45,46]$ and $\mathrm{Zr}[47,9$, 48]. In $\mathrm{Zn}$ and $\mathrm{Cd},\langle\mathbf{c}+\mathbf{a}\rangle$ dissociation on the Pyr. II plane is strongly favored over Pyr. I dissociation, indicating that the Pyr. II plane is the primary $\langle\mathbf{c}+\mathbf{a}\rangle$ slip plane, again consistent with experimental observations $[49,50,51,52,53]$. In contrast, the energy difference for $\mathrm{Mg}$ is small, with the MEAM material parameters marginally favoring Pyr. II while use of DFT SF data and experimental material properties indicates that Pyr. II is favorable. Direct atomistic measurements using the MEAM potential [33] also show Pyr. II dissociation is preferential with a total energy difference similar to the value predicted by MEAM data without core effects. In general, the small energy difference indicates that $\langle\mathbf{c}+\mathbf{a}\rangle$ slip can operate on both Pyr. I and II planes, but with Pyr. II slip likely to be dominant. For Mg, the close match between the elastic prediction and direct atomistic measurement again shows the core contribution is relatively small, indicating the robustness of the analysis.

Examining the individual contributions to the total energy difference shows that for $\mathrm{Ti}, \mathrm{Zr}, \mathrm{Zn}$ and $\mathrm{Cd}$, the interaction energy between the partials (green bar in Fig. 6b) plays the dominant role. The partial interaction energy depends on the energy prefactor $K_{12}$ and partial separation $r_{12}$. In these four metals, the variation of $K_{12}$ (yellow bar) between Pyr. I and II dissociations is relatively small, but $r_{12}$ varies significantly due to large variations in stacking fault energy $\gamma_{\mathrm{sf}}$. Therefore, $\gamma_{\mathrm{sf}}$ dictates the total energy difference in these four metals. For Mg (MEAM), $\mathrm{Mg}$, and $\mathrm{Re}$, the variation in $\gamma_{\mathrm{sf}}$ is small $(<30 \%)$. Energy contributions from the partial interaction and SF are larger than that from the angular term in these three metals. In addition, the effects of the angular term are different among different metals and slightly larger in $\mathrm{Zn}$ and $\mathrm{Cd}$. The latter two metals have highly anisotropic elastic properties. Overall, however, the effects of the angular term are relatively weak as compared to the effects in the mixed and edge $\langle\mathbf{c}+\mathbf{a}\rangle$ cases. This is expected, since for pure screw dislocations the angular term only arises from elastic anisotropy. In all cases, the total energy differences are nearly identical in the exact and approximate calculations. Similar to the previous two cases, the energy differences due to the core traction integral are relatively small and the dominant term is contained in the radial part of the partial interaction energy in the approximate calculations. 


\subsection{Basal - Prism - Pyramidal I screw $\langle\mathbf{a}\rangle$ transformations}

Figure $7 \mathrm{~b}$ shows the total energy difference and the associated contributing energies between a screw $\langle\mathbf{a}\rangle$ dislocation dissociated on Basal and Prism planes. Recall that the current DFT calculations do not show a metastable minimum for the Prism plane except in $\mathrm{Ti}, \mathrm{Zr}$ and $\mathrm{Re}$, so that for the other cases we use the unstable stacking fault energy as a proxy for a possible shallow metastable minimum. In $\mathrm{Mg}, \mathrm{Re}, \mathrm{Zn}$ and $\mathrm{Cd}$, the Basal plane is the energetically favorable dissociation plane for the screw $\langle\mathbf{a}\rangle$ dislocation, while in $\mathrm{Ti}$ and $\mathrm{Zr},\langle\mathbf{a}\rangle$ dissociation on the Prism plane, driven by its low SF energy, is strongly favored over the Basal plane. This is consistent with available experimental observations and earlier estimations [54] on the dominant $\langle\mathbf{a}\rangle$ slip plane in these metals [55].

Figure 7c shows the total energy difference and the associated contributing energies between a screw $\langle\mathbf{a}\rangle$ dislocation dissociated on the Prism and Pyr. I planes. In $\mathrm{Ti}$ and $\mathrm{Zr}$, the $\langle\mathrm{a}\rangle$ dissociated on the Prism plane has a slightly lower energy than that on the Pyr. I plane. In Zn and Cd, the Pyr. I-dissociated $\langle\mathbf{a}\rangle$ has a slightly lower energy as compared to dissociation on the Prism plane. In $\mathrm{Mg}(\mathrm{MEAM}), \mathrm{Mg}$ and Re, the energy difference between Prism and Pyr. I dissociations is marginal. In all metals, the $\langle\mathbf{a}\rangle$ dislocations have relatively small energy differences compared to the $\langle\mathbf{c}+\mathbf{a}\rangle$ dislocations in the previous three cases studied above. The respective energy contributions are comparable in $\mathrm{Mg}$ (MEAM), $\mathrm{Mg}, \mathrm{Ti}, \mathrm{Zr}$ and Re. The energy contribution due to partial interactions is dominant in $\mathrm{Zn}$ and $\mathrm{Cd}$, due to the large difference in the SF energies. Direct comparison with atomistic measurement is not possible in this case because the screw $\langle\mathbf{a}\rangle$ core structure dissociated on Pyr. I plane is not stable using the MEAM potential. We also note that the exact and approximate calculations show some appreciable differences in cases when the core separation $r_{12}$ is comparable to the core radii. Nevertheless, the absolute energy differences are small. In the cases when $r_{12} \rightarrow 2 r_{0}$ and the energy differences are small, direct atomistic simulations are needed for quantitative assessment.

\subsection{Pyramidal $\langle\mathbf{c}+\mathbf{a}\rangle \rightarrow\langle\mathbf{c}\rangle+\langle\mathbf{a}\rangle$}

The $\langle\mathbf{c}+\mathbf{a}\rangle$ dislocations on the Pyramidal planes could also be metastable against dissociation into separated $\langle\mathbf{c}\rangle$ and $\langle\mathbf{a}\rangle$, as supported by TEM observations [56], suggested by anisotropic elastic energy considerations [57] and demonstrated in previous MD simulations [6]. It is thus tempting to apply the present elastic analysis to these cases. However, it is not appropriate to do so. First and foremost, the dissociation of $\langle\mathbf{c}+\mathbf{a}\rangle \rightarrow\langle\mathbf{c}\rangle+\langle\mathbf{a}\rangle$ (see Fig. 8a and b) involves substantial core structure changes from $\langle\mathbf{c}+\mathbf{a}\rangle$ to $\langle\mathbf{c}\rangle$ and $\langle\mathbf{a}\rangle$, all of which are 

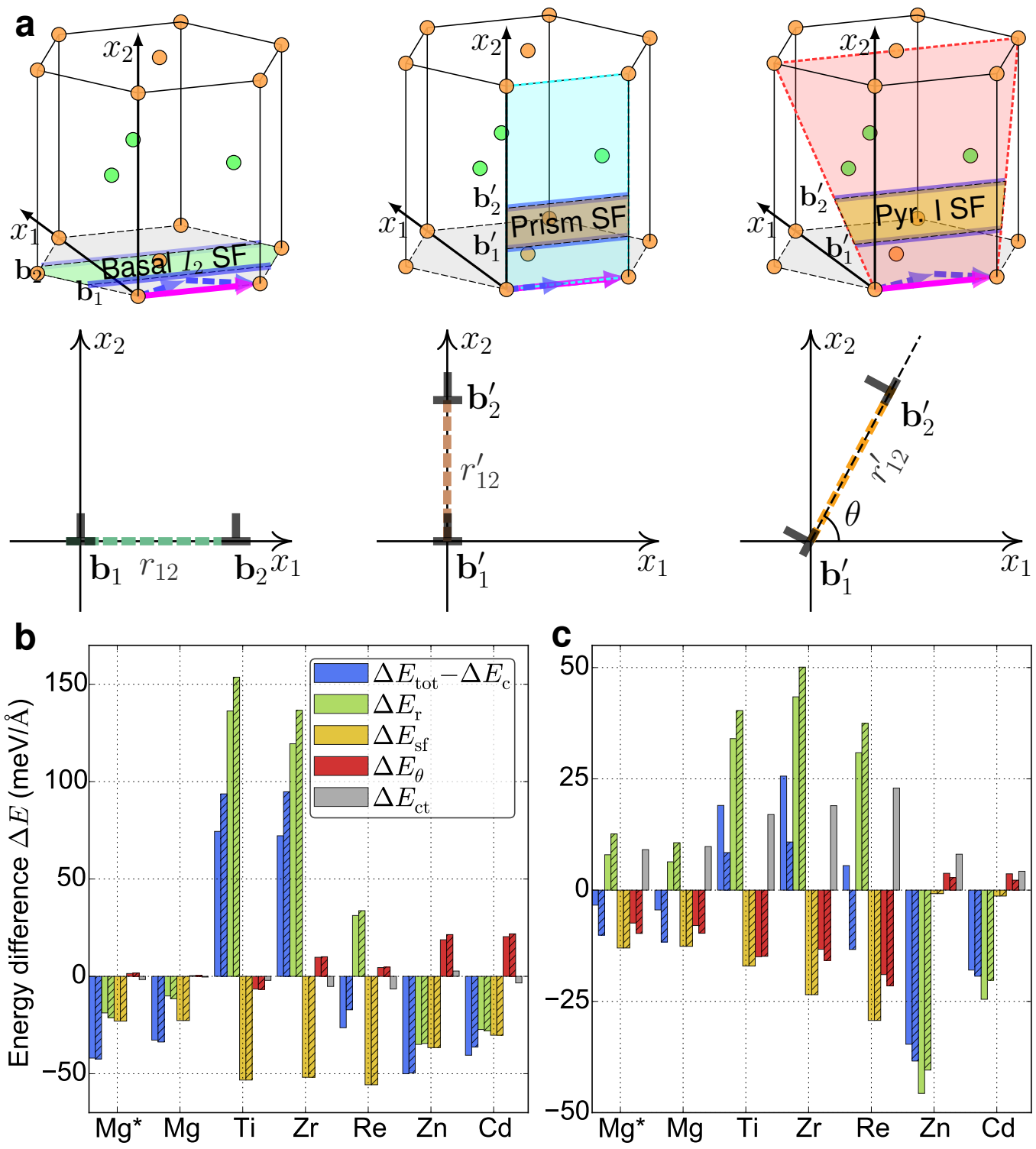

Figure 7: (a) Schematics and coordinate systems for screw $\langle\mathbf{a}\rangle$ dislocations dissociated on Basal, Prism and Pyramidal I planes. The total energy difference and contributing components are shown in (b) for Basal minus Prism and in (c) for Pyr. I minus Prism. In (b) and (c), Basal and Pyramidal I dissociation is energetically favorable when $\Delta E_{\text {tot }}-\Delta E_{\mathrm{c}}$ (the blue bar) is negative. $\mathrm{Mg}^{*}$ indicates the elastic predictions using the MEAM potential [33]. The hatched bars show the approximate results. The core radius of $\mathrm{Ti}$ is taken as $0.4 a$ due to its small dissociation separation on the Basal plane. The energy of Re is scaled by $1 / 3$. 
dissociated (and hence two partial dislocations are transformed into four partial dislocations), suggesting substantial changes in core energy; such a problem is thus outside the scope here. Second, MD simulations of Mg show that the $\langle\mathbf{c}\rangle$ dislocation itself undergoes spontaneous climb dissociation into a structure dissociated on the Basal plane, with an extrinsic stacking fault; hence the relevant $\langle$ c $\rangle$ structure is not clear. Third, MD simulations on Mg show that the widelyseparated $\langle\mathbf{c}\rangle$ and $\langle\mathbf{a}\rangle$ are higher in energy than the $\langle\mathbf{c}+\mathbf{a}\rangle$ dissociated on the Basal plane; this makes dissociation unlikely in the absence of applied stresses. Fourth, MD simulations on $\mathrm{Mg}$, which include all core energies and interactions, also show that there are close-core interactions between the $\langle\mathbf{c}\rangle$ and $\langle\mathbf{a}\rangle$ dislocations that make the close-core case lower in energy than the widely separated $\langle\mathbf{c}\rangle$ and $\langle\mathbf{a}\rangle$. Quantitative analysis of $\langle\mathbf{c}+\mathbf{a}\rangle \rightarrow\langle\mathbf{c}\rangle+\langle\mathbf{a}\rangle$ dissociations is thus intricate and relevant cases will generally involve core changes and/or core interactions, making the problem outside the scope of the present paper.

Nevertheless, when the separation between the $\langle\mathbf{c}\rangle$ and $\langle\mathbf{a}\rangle$ is large compared to their individual core dissociation distances, the elastic interaction energy between them can be approximated by treating them as compact full dislocations, as shown in Fig. 8c. Studies in Refs. $[58,59,1]$ show that $K_{12}$ in Eq. 14 is zero, i.e. the radial part of the elastic interaction energy is zero, while the work of Yoo [57] seems to claim $K_{12} \neq 0$. The fact that $K_{12}=0$ is (i) verified by our explicit calculations using the elastic formalism of Stroh given earlier, (ii) expected physically because a nonzero $K_{12}$ would lead to a divergent energy (calculations of $K, K_{1}$ and $K_{2}$ show that $K=K_{1}+K_{2}$ ), and (iii) verified by explicit atomistic measurement in $\mathrm{Mg}$ where $K$ of the $\langle\mathbf{c}+\mathbf{a}\rangle$ on the Pyr. II plane is exactly the sum of $K$ of a standalone $\langle\mathbf{c}\rangle$ and $\langle\mathbf{a}\rangle$. Thus, only the angular part of the interaction energy $E_{\theta}$ is non-zero, which is independent of the dislocation separation $r_{12}$. Figure $8 \mathrm{~d}$ and e show $E_{\theta}$ for the Pyr. I mixed and Pyr. II edge orientations (see Figure 8a and b), respectively. The interaction energy is $-E_{\theta}$ and so there are preferential orientations of well-separated $\langle\mathbf{c}\rangle$ and $\langle\mathbf{a}\rangle$ dislocations corresponding to the maxima of $E_{\theta}$. In $\mathrm{Mg}$, simulations show that dissociation of dissociated $\langle\mathbf{c}\rangle$ and $\langle\mathbf{a}\rangle$ remaining in close proximity can have $\theta$ deviating from zero, leading to a lowering of the energy. Dissociation accompanied by glide of the $\langle\mathbf{c}\rangle$ and $\langle\mathbf{a}\rangle$ on the Prism and Basal planes could also lead to a non-zero $\theta$, particularly at small separations, and hence a lowering of the total energy. Glide of the $\langle\mathbf{c}\rangle$ on the Prism plane is unlikely, however, since analyses indicate that basal-climb dissociation of the $\langle\mathbf{c}\rangle$ is highly likely in most of the hcp metals considered here. For a general pair of $\langle\mathbf{c}\rangle$ and $\langle\mathbf{a}\rangle$ existing in the lattice, the variation of the angular energy will lead to forces on the $\langle\mathbf{a}\rangle$ acting to drive it to glide along the Basal or Prism plane 


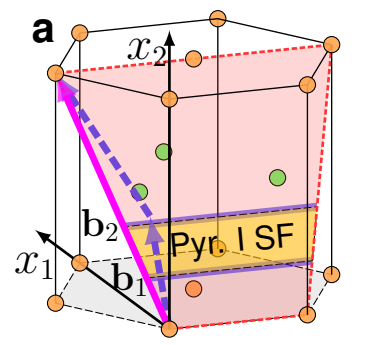

$$
\langle\mathbf{c}+\mathbf{a}\rangle
$$
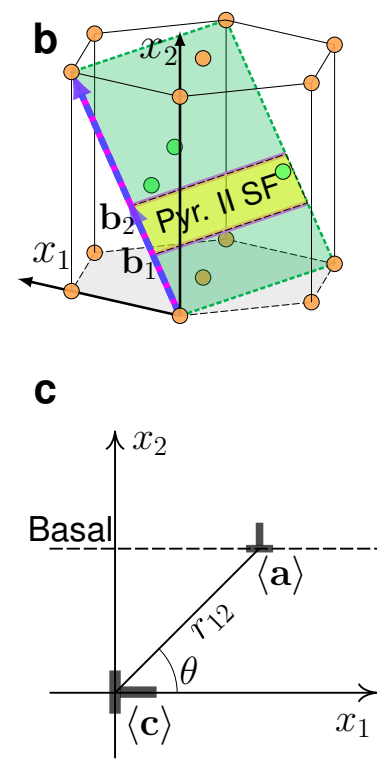

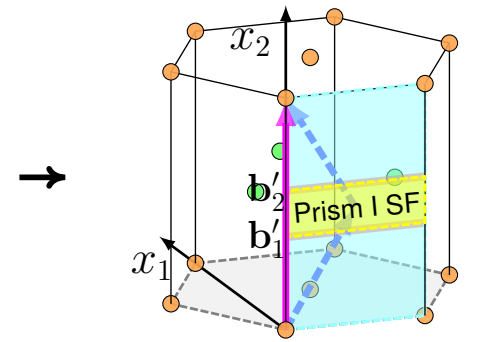

$\langle\mathbf{c}\rangle$
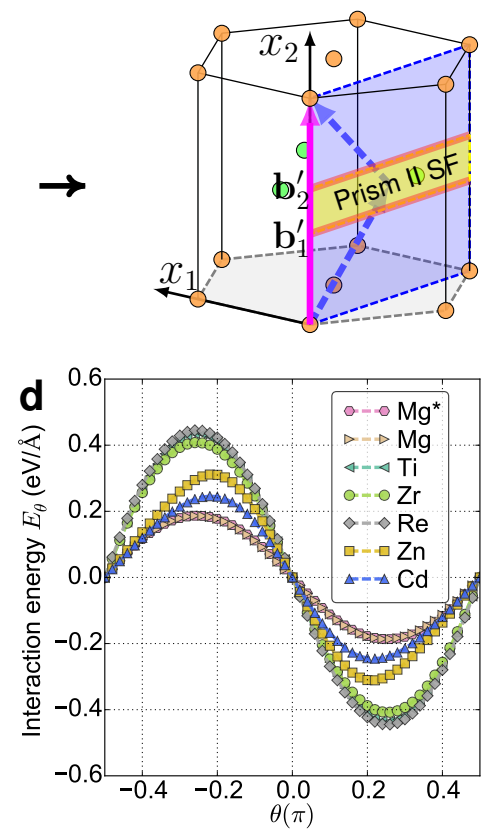

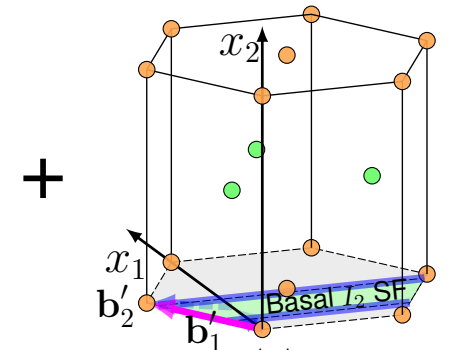

$\langle\mathbf{a}\rangle$
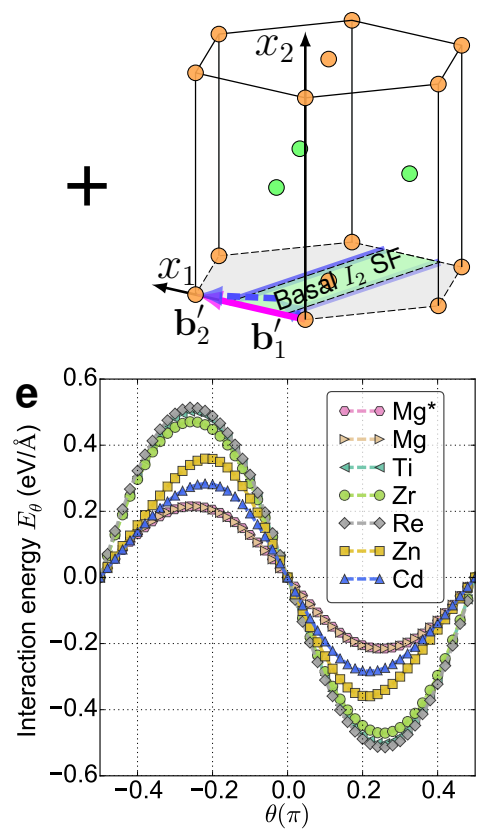

Figure 8: Schematics and coordinate systems for $\langle\mathbf{c}+\mathbf{a}\rangle$ dislocations dissociated on (a) Pyramidal I and (b) II planes transform into $\langle\mathbf{c}\rangle$ and $\langle\mathbf{a}\rangle$ dislocations. The $\langle\mathbf{c}\rangle$ core dissociations on Prism planes are shown for illustrations only and Basal climb-dissociations are highly likely. (c) Coordinate system for interaction between the undissociated edge $\langle\mathbf{c}\rangle$ and $\langle\mathbf{a}\rangle$ dislocations. (d-e) Angular component of the interaction energy as a function of $\theta$ for the Pyramidal I mixed and Pyramidal II edge orientation. Mg* indicates the elastic predictions using the MEAM potential [33]. The energy of Re is scaled by $1 / 3$. 
to the energetically-favorable $\theta$. Applied forces would then be necessary to move the $\langle\mathbf{a}\rangle$ dislocation away from that energetically-favorable $\theta$.

\section{Discussion}

A broad range of experiments show long, straight $\langle\mathbf{c}+\mathbf{a}\rangle$ dislocation segments with Basal orientations in the family of hcp metals [42, 60, 61, 62, 11, 63, 28, 64, 65]. The preferential Basal orientations could be due to intrinsic high Peierls stresses of $\langle\mathbf{c}+\mathbf{a}\rangle$ dislocations in the close-packed Basal orientations, to $\langle\mathbf{c}+\mathbf{a}\rangle$ climb-dissociation to the Basal plane, or a combination of the two. However, even if the long straight segments were initially confined by a high Peierls stress, such dislocations would very likely still undergo climb dissociation due to the large reduction in energy even at nanoscale climb distances. For Mg, previous MD simulations [6, 7], experimental observations $[66,65]$, and the present elastic analysis all suggest that the $\langle\mathbf{c}+\mathbf{a}\rangle$ Pyr. II dislocation undergoes climb-dissociation onto the Basal plane. For the other hcp metals, experiments show that, at high temperatures, Basal-oriented $\langle\mathbf{c}+\mathbf{a}\rangle$ dislocations are observed more frequently in Ti $[42,62]$ and Ti-36.5at.\%Al [11], while $\langle\mathbf{c}+\mathbf{a}\rangle$ slip becomes increasingly difficult in Ti-6.6Al [10]. These observations suggest a thermally-activated process governing the $\langle\mathbf{c}+\mathbf{a}\rangle$ slip, and so the long straight segments along the Pyr. II edge or Pyr. I mixed line direction may be the result of precisely the climb dissociation process analyzed here and demonstrated in $\mathrm{Mg}$ MD simulations.

For the $\langle\mathbf{c}+\mathbf{a}\rangle$ screw dislocations, the elastic analysis shows that for $\mathrm{Mg}$ (MEAM), the Pyr. II dissociation has 3-4 meV/A lower energy than the Pyr. I dissociation, while direct atomistic measurement shows Pyr. II dissociation has a lower energy, $\sim 3 \mathrm{meV} / \AA$, close to the prediction using properties of the MEAM potential. The prediction using experimental and DFT data shows larger energy difference, $\sim 30 \mathrm{meV} / \AA$ more favorable on the Pyr. II plane. We attribute the discrepancy between Mg (MEAM) and DFT to the differences in SF energy on the Pyramidal II plane. In addition, while the elastic prediction here is in remarkable agreement with the direct atomistic measurement, we shall still emphasize that the dislocation core energy may not be precisely captured in the MEAM potential and in real materials, the core energy differences may still be appreciable. Energy differences on the order of $10 \mathrm{~s}$ of meV/A are beyond the scope of our analysis, since such energies can be, and evidently are (for $\mathrm{Mg}$ ), on the scale of core-energy differences. Nevertheless, a small energy difference predicted by the analysis has a few implications. First, when the elastic analysis predicts small energy differences $(10 \mathrm{~s}$ of $\mathrm{meV} / \AA$ ) between configurations, this is an indication that direct DFT 
computations of core structures with appropriate boundary conditions are needed to resolve the differences with the accuracy needed to make even qualitative predictions. Second, small energy differences indicate relatively easy cross-slip, as we have revealed in transition path calculations of $\langle\mathbf{c}+\mathbf{a}\rangle$ cross-slip in $\mathrm{Mg}$ [15]. The cross-slip energy barrier is directly related to the energy difference, which can be overcome by applied shear stresses on the cross-slip plane. A small energy difference also suggests that other factors may influence the core and SF energies, and thus the relative energy balance. Applied stresses and/or solid solution alloying, through interactions with the dislocation core and stacking fault, can thus shift the dislocation energy difference, the relative stability, and the frequency of cross-slip. The energy differences estimated from Mg (MEAM) are quite small and we have recently shown that such energy differences can be changed by applied stresses, leading to relatively easy cross-slip or even a switch of the stable slip plane for the $\langle\mathbf{c}+\mathbf{a}\rangle$ dislocations [15]. It is thus difficult to determine the primary $\langle\mathbf{c}+\mathbf{a}\rangle$ slip plane in experiments [26, 27, 28, 67] and to precisely reproduce the true $\langle\mathbf{c}+\mathbf{a}\rangle$ slip behavior in dislocation modeling at all scales. For $\mathrm{Ti}$, $\mathrm{Zr}, \mathrm{Re}, \mathrm{Zn}$ and $\mathrm{Cd}$, the analysis shows clear distinctions between Pyr. I and II plane dissociation. In these metals, the large energy differences suggest $\langle\mathbf{c}+\mathbf{a}\rangle$ slip should dominate on the predicted Pyramidal plane and cross-slip should be difficult. However, some experiments in Ti and its alloys show cross-slip [68, 30] and Pyr. II $\langle\mathbf{c}+\mathbf{a}\rangle$ slip [62, 69, 10, 70, 71], particularly in c-axis compression tests at high temperatures. In these experiments, Pyr. II $\langle\mathbf{c}+\mathbf{a}\rangle$ slip and cross-slip may be activated by stress, temperature, solid solution alloying, or a combination of them. We also expect the contribution of core energy difference in Ti is stronger than in $\mathrm{Mg}$, which may further reduce the predicted energy difference. Future direct atomistic simulations of $\langle\mathbf{c}+\mathbf{a}\rangle$ core in $\mathrm{Ti}$ are thus desirable, given its high technological value.

For the screw $\langle\mathbf{a}\rangle$ dislocation, the elastic analysis shows clear differences between Basal and Prism dissociations and small energy differences between Prism and Pyr. I dissociations in all 6 metals. For $\mathrm{Mg}, \mathrm{Re}, \mathrm{Zn}$ and $\mathrm{Cd}$, our calculations indicate that the primary slip plane for $\langle\mathbf{a}\rangle$ dislocations is the Basal plane, given its very low SF energy relative to that on Prism and Pyr. I planes. Dissociation of Basal $\langle\mathbf{a}\rangle$ dislocations is similar to that in face-centered cubic metals, with partials of mixed character, so that cross-slip out of the Basal plane is difficult, as is well-known. Therefore, $\langle\mathbf{a}\rangle$ slip is dominant on Basal planes in these metals, even though $\langle\mathbf{a}\rangle$ slip on Prism and Pyr. I planes is still possible, as seen in previous experiments [55]. For Ti, our analysis shows the energy for Prism dissociation is $\sim 19 \mathrm{meV} / \AA$ lower than that for Pyr. I dissociation. This is close to the value 
$\sim 4 \mathrm{meV} / \mathrm{A}$ measured in DFT calculations with flexible boundary conditions [72] and the value $-5.7 \mathrm{meV} / \AA$ measured in DFT calculations using periodic boundary conditions [5]. For $\mathrm{Zr}$, our analysis shows Prism dissociation is $\sim 26 \mathrm{meV} / \AA$ lower than Pyr. I dissociation, in contrast to $\sim 2.9 \mathrm{meV} / \AA$ measured in DFT with periodic boundary conditions [5]. While these different calculations show discrepancies in the quantitative values, all results suggest the energy difference is small and may be changed by other factors, as in the case of screw $\langle\mathbf{c}+\mathbf{a}\rangle$ dislocations. Again, these energy differences are obviously beyond the accuracy of the current linear elastic framework.

In summary, we have studied the energetics of dislocation dissociations based on anisotropic linear elastic theory and stacking fault energies. Using appropriate elastic constants, stacking fault energies, and dislocation Burgers vectors, this single framework is able to capture the essential differences among the different dissociations and slip systems, and among different hcp metals. The elastic analysis here is robust and provides a baseline assessment of the fundamental dislocation energy, dissociations, and associated primary slip systems across the family of hcp metals. The results obtained not only rationalize broad experimental observations on dominant slip systems and dislocation behaviour, but also provide predictions for possible dislocation dissociations in hcp metals. We note again that the current analysis does not take into account the core energy contribution nor does it provide the energy barrier and kinetics for transitions between the different dissociated configurations. It thus remains essential for future work, such as high resolution TEM, to validate the predictions further. Direct atomistic simulations with appropriate interatomic potentials or DFT calculations with appropriate boundary conditions are also useful to reveal the transition path and/or energetic information under more complicated conditions, such as stress, temperature or solid-solution alloying.

\section{Acknowledgements}

The authors acknowledge financial support of this work through a grant from the Swiss National Science Foundation entitled "Control of Atomistic Mechanisms of Flow in Magnesium Alloys to Achieve High Ductility" (project \#162350), as well as computer time supported by a grant from the Swiss National Supercomputing Centre (CSCS) under project ID s631. Z.W. further acknowledges the financial support from the Agency for Science, Technology and Research (A*STAR), Singapore. 


\section{Appendix. Elastic energy of an arbitrary dislocation pair}

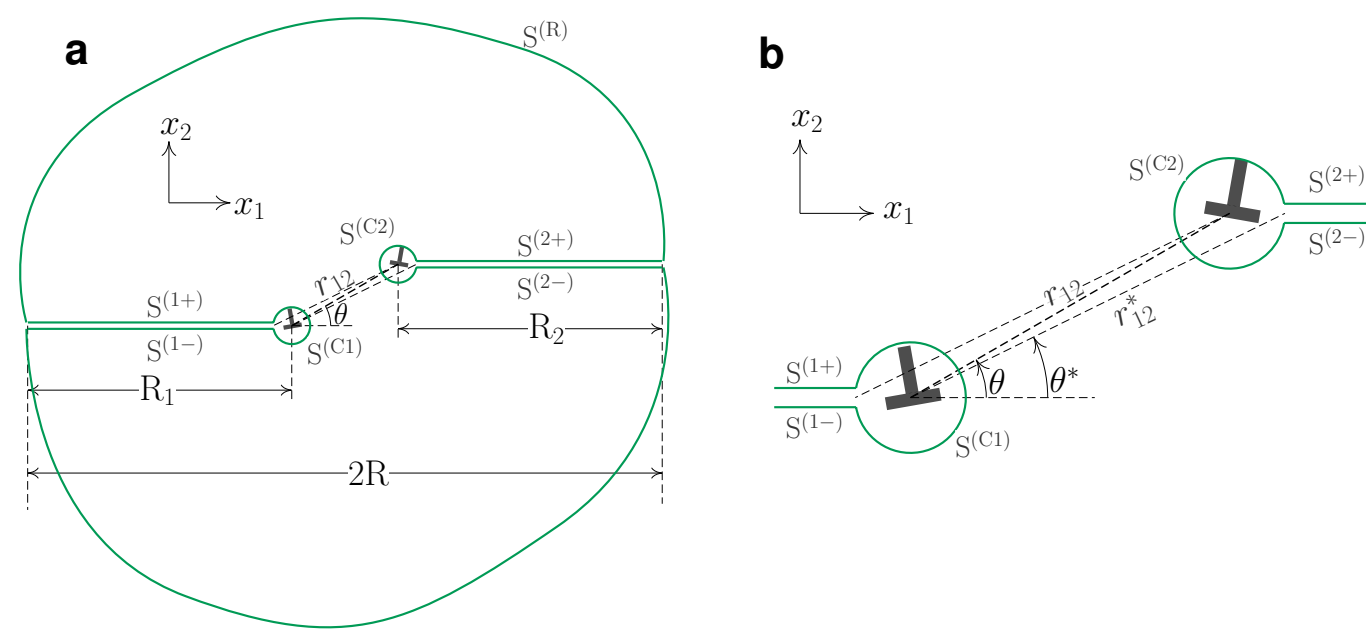

Figure A.1: (a) A pair of dislocations $\mathbf{b}_{1}$ and $\mathbf{b}_{2}$ with line direction $\boldsymbol{\xi}$ in $x_{3}$ in an arbitrary domain. The two dislocations are separated by $\left(r_{12}, \theta\right)$. $\mathrm{S}^{(1 \pm)}$ and $\mathrm{S}^{(2 \pm)}$ are the two cut planes along the $x_{1}$ axis and $\mathrm{S}^{(\mathrm{C} 1)}$ and $\mathrm{S}^{(\mathrm{C} 2)}$ are the circular paths enclosing the dislocation cores. $R_{1} \approx R_{2} \approx R \gg$ $r_{12}$ and $\mathrm{S}^{(\mathrm{R})}$ is the path enclosing the arbitrary area which can be made arbitrarily large. The total energy is independent of the choice of the cut planes. (b) The same pair of dislocations in close view.

We calculate the total energy of an arbitrary pair of dislocations using the general surface traction integral method. We consider two dislocations with Burgers vectors $\mathbf{b}_{1}$ and $\mathbf{b}_{2}$ and line direction $\boldsymbol{\xi}$ in $x_{3}$, as shown in Fig. A.1. The elastic energy per unit length inside an arbitrary area and excluding the two dislocation core areas can be found by integrating the strain energy density within the volume as

$$
E_{\mathrm{e}}=\frac{1}{2} \int_{V}\left(\boldsymbol{\sigma}^{(1)}+\boldsymbol{\sigma}^{(2)}\right):\left(\boldsymbol{\varepsilon}^{(1)}+\boldsymbol{\varepsilon}^{(2)}\right) \mathrm{d} V
$$

The above integral is equivalent to the work done by the traction over the surface enclosing the volume as

$$
E_{\mathrm{e}}=\frac{1}{2} \int_{\Omega}\left(\mathbf{u}^{(1)}+\mathbf{u}^{(2)}\right)^{\mathrm{T}} \cdot\left(\boldsymbol{\sigma}^{(1)}+\boldsymbol{\sigma}^{(2)}\right) \cdot \mathbf{n} \mathrm{d} s
$$

where the integration is carried out along the path enclosing the volume $V$ and excluding any discontinuity and singularity in $\mathbf{u}$ and $\boldsymbol{\sigma}$, i.e., along $\mathrm{S}^{(\mathrm{C} 1)}, \mathrm{S}^{(1 \pm)}$, 
$\mathrm{S}^{(\mathrm{R})}, \mathrm{S}^{(2 \pm)}$ and $\mathrm{S}^{(\mathrm{C} 2)}$, (see Fig. A.1a). The total elastic energy is thus the sum of 5 path integrals as

$$
E_{\mathrm{e}}=E_{\mathrm{e}}^{\mathrm{S}^{(\mathrm{C} 1)}}+E_{\mathrm{e}}^{\mathrm{S}^{(\mathrm{C} 2)}}+E_{\mathrm{e}}^{\mathrm{S}^{(1 \pm)}}+E_{\mathrm{e}}^{\mathrm{S}^{(2 \pm)}}+E_{\mathrm{e}}^{\mathrm{S}^{(\mathrm{R})}}
$$

As in the case of a single dislocation, the total elastic energy is again independent of the choice of the cut plane. However, in the above equation, each individual integral is cut-plane-dependent. For a consistent evaluation of the total dislocation energy, all terms have to be included and computed precisely in the calculation.

On path $\mathrm{S}^{(\mathrm{C} 1)}$

$$
E_{\mathrm{e}}^{\mathrm{S}^{(\mathrm{C} 1)}}=\frac{1}{2} \int_{\mathrm{S}^{(\mathrm{C} 1)}}\left[u_{i}^{(1)} \sigma_{i j}^{(1)}+u_{i}^{(1)} \sigma_{i j}^{(2)}+u_{i}^{(2)} \sigma_{i j}^{(1)}+u_{i}^{(2)} \sigma_{i j}^{(2)}\right] n_{j} \mathrm{~d} s
$$

The first term in the above equation is the self core traction of dislocation $\mathbf{b}_{1}$ in an infinite domain. The integral was obtained analytically in isotropic media by Bullough and Foreman [24] and in anisotropic media by Clouet [18]. The last three terms become small when the dislocation separation $r_{12}$ is large compared to the core cut off radius $r_{01}$. The integral on path $\mathrm{S}^{(\mathrm{C} 2)}$ is similar,

$$
E_{\mathrm{e}}^{\mathrm{S}^{(\mathrm{C} 2)}}=\frac{1}{2} \int_{\mathrm{S}^{(\mathrm{C} 2)}}\left[u_{i}^{(1)} \sigma_{i j}^{(1)}+u_{i}^{(1)} \sigma_{i j}^{(2)}+u_{i}^{(2)} \sigma_{i j}^{(1)}+u_{i}^{(2)} \sigma_{i j}^{(2)}\right] n_{j} \mathrm{~d} s
$$

where the last term is the self core traction of dislocation $\mathbf{b}_{2}$ in an infinite domain. On path $\mathrm{S}^{(1 \pm)}$,

$$
\begin{aligned}
E_{\mathrm{e}}^{\mathrm{S}^{(1 \pm)}} & =\frac{1}{2} \int_{\mathrm{S}^{(1 \pm)}}\left[u_{i}^{(1)} \sigma_{i j}^{(1)}+u_{i}^{(1)} \sigma_{i j}^{(2)}+u_{i}^{(2)} \sigma_{i j}^{(1)}+u_{i}^{(2)} \sigma_{i j}^{(2)}\right] n_{j} \mathrm{~d} s \\
& =\frac{1}{2} \int_{\mathrm{S}^{(1 \pm)}} u_{i}^{(1)} \sigma_{i j}^{(1)} n_{j} d s+\frac{1}{2} \int_{\mathrm{S}^{(1 \pm)}} u_{i}^{(1)} \sigma_{i j}^{(2)} n_{j} \mathrm{~d} s
\end{aligned}
$$

The first integral is the same as that of the elastic self-energy of $\mathbf{b}_{1}$ as shown in Eq. 9. The second integral is similar to the interaction energy of dislocation $\mathbf{b}_{1}$ and $\mathbf{b}_{2}$, as shown in Eq. 13 and 14. Therefore, Eq. A.6 reduces to

$$
\begin{aligned}
E_{\mathrm{e}}^{\mathrm{S}^{(1 \pm)}} & =K_{1} \ln \frac{R_{1}}{r_{01}}+\frac{1}{2} K_{12} \ln \frac{R_{1}+r_{12} \cos \theta}{r_{12}^{*}}-\frac{1}{2} E_{\theta^{*}} \\
& \simeq K_{1} \ln \frac{R}{r_{01}}+\frac{1}{2} K_{12} \ln \frac{R}{r_{12}^{*}}-\frac{1}{2} E_{\theta^{*}}, \text { when } R_{1} \approx R \gg r_{12}
\end{aligned}
$$


where $R_{1}$ is the distance from the outer boundary to the core of $\mathbf{b}_{1}$ (see Fig. A.1a and $b$ ), and

$$
\begin{aligned}
r_{12}^{*} & =\sqrt{\left(r_{12} \sin \theta\right)^{2}+\left(r_{12} \cos \theta+r_{0 i}\right)^{2}} \\
\theta^{*} & =\arctan \frac{r_{12} \sin \theta}{r_{12} \cos \theta+r_{0 i}}
\end{aligned}
$$

$K_{1}$ is the energy prefactor of dislocation $\mathbf{b}_{1}, K_{12}$ is the interaction energy prefactor of dislocations $\mathbf{b}_{1}$ and $\mathbf{b}_{2}, E_{\theta^{*}}$ is the angular dependent part of the interaction energy, all as found earlier in Eq. 9 and 14. Similarly, on path $\mathrm{S}^{2 \pm}$,

$$
\begin{aligned}
E_{\mathrm{e}}^{\mathrm{S}^{2 \pm}} & =\frac{1}{2} \int_{\mathrm{S}^{(2 \pm)}}\left[u_{i}^{(1)} \sigma_{i j}^{(1)}+u_{i}^{(1)} \sigma_{i j}^{(2)}+u_{i}^{(2)} \sigma_{i j}^{(1)}+u_{i}^{(2)} \sigma_{i j}^{(2)}\right] n_{j} \mathrm{~d} s \\
& =\frac{1}{2} \int_{\mathrm{S}^{(2 \pm)}} u_{i}^{(2)} \sigma_{i j}^{(2)} n_{j} d s+\frac{1}{2} \int_{\mathrm{S}^{(2 \pm)}} u_{i}^{(2)} \sigma_{i j}^{(1)} n_{j} \mathrm{~d} s
\end{aligned}
$$

The first integral is the same as that of the elastic self-energy of $\mathbf{b}_{2}$. The second integral is similar to the interaction energy of dislocation $\mathbf{b}_{1}$ and $\mathbf{b}_{2}$. Therefore, Eq. A.9 reduces to

$$
\begin{aligned}
E_{\mathrm{e}}^{\mathrm{S}^{2 \pm}} & =K_{2} \ln \frac{R_{2}}{r_{02}}+\frac{1}{2} K_{12} \ln \frac{R_{2}+r_{12} \cos \theta}{r_{12}^{*}}-\frac{1}{2} E_{\theta^{*}} \\
& \simeq K_{2} \ln \frac{R}{r_{02}}+\frac{1}{2} K_{12} \ln \frac{R}{r_{12}^{*}}-\frac{1}{2} E_{\theta^{*}}, \text { when } R_{2} \approx R \gg r_{12}
\end{aligned}
$$

In both Eq. A.7 and A.10, $r_{12}^{*} \rightarrow r_{12}$ and $\theta^{*} \rightarrow \theta$ when the core radii $r_{0 i} \rightarrow 0$ or $r_{12}$ is large compared to $r_{0 i}$ (see Fig. A.1b), i.e. Eq. A.7 and A.10 approach half of the interaction energy of dislocation $b_{1}$ and $b_{2}$ as computed in Eq. 14. When the core radii $r_{0 i}$ are taken as finite, but $r_{12}$ is large compared to $r_{0 i}$, both (i) the sum of the second integral in Eq. A.6 and the second integral in Eq. A.4 and (ii) the sum of the second integral in Eq. A.9 and the third integral in Eq. A.5 approach half of the interaction energy of dislocation $b_{1}$ and $b_{2}$ as computed in Eq. 14. The two terms (the second integral in Eq. A.4 and the third integral in Eq. A.5) in the traction integral at the two cores are thus contained in the radial interaction energy in Eq. 14 of the approximate formulation.

Finally, the integral along the path $S^{(R)}$ in the far field is

$$
E_{\mathrm{e}}^{\mathrm{S}^{(\mathrm{R})}}=\frac{1}{2} \int_{\mathrm{S}^{(\mathrm{R})}}\left[u_{i}^{(1)} \sigma_{i j}^{(1)}+u_{i}^{(1)} \sigma_{i j}^{(2)}+u_{i}^{(2)} \sigma_{i j}^{(1)}+u_{i}^{(2)} \sigma_{i j}^{(2)}\right] n_{j} \mathrm{~d} s
$$


When $R \gg r_{12}$, the above integral (with the cut plane along the $x_{1}$ axis) is equivalent to that of a single dislocation with Burgers vector $\mathbf{b}_{1}+\mathbf{b}_{2}$, i.e. the integral over the far field path only depends on the total Burgers vector and is independent of the details of the individual dislocations.

Substituting Eq. A.4, A.5, A.7, A.10 and A.11 into the total elastic energy in Eq. A.3 leads to Eq. 19 in Section 2.3. In the current work, we evaluate all terms precisely and compare the exact results with the approximate ones. In all our cases, the energy differences based on the two approaches are negligible within the accuracy of linear elastic framework.

\section{References}

[1] J. P. Hirth, J. Lothe, Theory of Dislocations, 2 ed., John Wiley \& Sons, 1982.

[2] B. H. Kear, H. G. F. Wilsdorf, Dislocation configurations in plastically deformed polycrystalline $\mathrm{Cu}_{3} \mathrm{Au}$ alloys, Transactions of the Metallurgical Society of AIME 224 (1962) 382-386.

[3] V. Paidar, D. P. Pope, V. Vitek, A theory of the anomalous yield behavior in $\mathrm{L}_{2}$ ordered alloys, Acta Metallurgica 32 (1984) 435-448.

[4] V. Vitek, D. P. Pope, J. L. Bassani, Anomalous yield behaviour of compounds with $\mathrm{L}_{2}$ structure, Dislocations in Solids 10 (1996) 135-185.

[5] E. Clouet, D. Caillard, N. Chaari, F. Onimus, D. Rodney, Dislocation locking versus easy glide in titanium and zirconium, Nature Materials 14 (2015) 931-936.

[6] Z. Wu, W. A. Curtin, The origins of high hardening and low ductility in magnesium, Nature 526 (2015) 62-67.

[7] Z. Wu, W. A. Curtin, Intrinsic structural transitions of the pyramidal I $\langle c+a\rangle$ dislocation in magnesium, Scripta Materialia 116 (2016) 104-107.

[8] H. Numakura, Y. Minonishi, M. Koiwa, $\langle\overline{1} \overline{1} 23\rangle\{10 \overline{1} 1\}$ slip in titanium polycrystals at room temperature, Scripta Metallurgica 20 (1986) 1581-1586.

[9] H. Numakura, Y. Minonishi, M. Koiwa, $\langle\overline{1} \overline{1} 23\rangle\{10 \overline{1} 1\}$ slip in zirconium, Philosophical Magazine A 63 (1991) 1077-1084. 
[10] J. C. Williams, R. G. Baggerly, N. E. Paton, Deformation behavior of HCP Ti-Al alloy single crystals, Metallurgical and Materials Transactions A Physical Metallurgy and Materials Science 33 (2002) 837-850.

[11] H. Inui, Y. Toda, Y. Shirai, M. Yamaguchi, Low-temperature deformation of single crystals of a DO19 compound with an off-stoichiometric composition (ti-36.5 at.\% al), Philosophical Magazine A 69 (1994) 1161-1177.

[12] J. Eshelby, W. Read, W. Shockley, Anisotropic elasticity with applications to dislocation theory, Acta Metallurgica 1 (1953) 251-259.

[13] A. N. Stroh, Dislocations and cracks in anisotropic elasticity, Philosophical Magazine 3 (1958) 625-646.

[14] T. C. Ting, Anisotropic Elasticity: Theory and Applications, Oxford University Press, 1996.

[15] Z. Wu, W. A. Curtin, Mechanism and energetics of $\langle c+a\rangle$ dislocation crossslip in hcp metals, Proceedings of the National Academy of Sciences of the United States of America (2016) submitted.

[16] V. Vitek, Intrinsic stacking faults in body-centred cubic crystals, Philosophical Magazine 18 (1968) 773-786.

[17] D. J. Bacon, D. M. Barnett, R. O. Scattergood, Anisotropic continuum theory of lattice defects, Progress in Materials Science 23 (1980) 51-262.

[18] E. Clouet, Elastic energy of a straight dislocation and contribution from core tractions, Philosophical Magazine 89 (2009) 1565-1584.

[19] Z. Wu, W. A. Curtin, Brittle and ductile crack-tip behavior in magnesium, Acta Materialia 88 (2015) 1-12.

[20] A. H. Cottrell, Theory of dislocations, Progress in Metal Physics 1 (1949) 77-96.

[21] F. R. N. Nabarro, Mathematical theory of stationary dislocations, Advances in Physics 1 (1952) 269-394.

[22] M. Peach, J. S. Koehler, The forces exerted on dislocations and the stress fields produced by them, Physical Review 80 (1950) 436-439. 
[23] M. H. Yoo, On the theory of anomalous yield behavior of $\mathrm{Ni}_{3} \mathrm{Al}$-effect of elastic anisotropy, Scripta Metallurgica 20 (1986) 915-920.

[24] R. Bullough, E. A. J. Foreman, The elastic energy of a rhombus-shaped dislocation loop, Philosophical Magazine 9 (1964) 315-329.

[25] S. R. Agnew, L. Capolungo, C. A. Calhoun, Connections between the basal I1 "growth" fault and $\langle c+a\rangle$ dislocations, Acta Materialia 82 (2015) 255265.

[26] H. Fan, J. A. El-Awady, Towards resolving the anonymity of pyramidal slip in magnesium, Materials Science and Engineering: A 644 (2015) 318-324.

[27] K. Y. Xie, Z. Alam, A. Caffee, K. J. Hemker, Pyramidal I slip in c-axis compressed Mg single crystals, Scripta Materialia 112 (2016) 75-78.

[28] S. Sandlöbes, M. Friák, J. Neugebauer, D. Raabe, Basal and non-basal dislocation slip in Mg-Y, Materials Science and Engineering: A 576 (2013) 61-68.

[29] S. R. Agnew, D. W. Brown, C. N. Tomé, Validating a polycrystal model for the elastoplastic response of magnesium alloy AZ31 using in situ neutron diffraction, Acta Materialia 54 (2006) 4841-4852.

[30] R. Ding, J. Gong, A. J. Wilkinson, I. P. Jones, $\langle c+a\rangle$ dislocations in deformed Ti-6Al-4V micro-cantilevers, Acta Materialia 76 (2014) 127-134.

[31] C. S. Barrett, T. B. Massalski, Structure of Metals, International Series on Materials Science and Technology, 3 ed., Pergamon, 1980.

[32] G. Simmons, H. Wang, Single Crystal Elastic Constants and Calculated Aggregate Properties. A Handbook, 2 ed., The MIT Press, 1971.

[33] Z. Wu, M. F. Francis, W. A. Curtin, Magnesium interatomic potential for simulating plasticity and fracture phenomena, Modelling and Simulation in Materials Science and Engineering 23 (2015) 015004.

[34] G. Kresse, J. Furthmüller, Efficient iterative schemes for ab initio totalenergy calculations using a plane-wave basis set, Physical Review B 54 (1996) 11169-11186. 
[35] G. Kresse, D. Joubert, From ultrasoft pseudopotentials to the projector augmented-wave method, Physical Review B 59 (1999) 1758-1775.

[36] J. P. Perdew, K. Burke, M. Ernzerhof, Generalized gradient approximation made simple, Physical Review Letters 77 (1996) 3865-3868.

[37] P. E. Blöchl, Projector augmented-wave method, Physical Review B 50 (1994) 17953-17979.

[38] J. R. Morris, J. Scharff, K. M. Ho, D. E. Turner, Y. Y. Ye, M. H. Yoo, Prediction of a $\{11 \overline{2} 2\}$ hcp stacking fault using a modified generalized stackingfault calculation, Philosophical Magazine A 76 (1997) 1065-1077.

[39] N. Chaari, E. Clouet, D. Rodney, First order pyramidal slip of $1 / 3<1 \overline{2} 10>$ screw dislocations in zirconium, Metallurgical and Materials Transactions A 45 (2014) 5898-5905.

[40] P. Kwasniak, H. Garbacz, K. J. Kurzydlowski, Solid solution strengthening of hexagonal titanium alloys: Restoring forces and stacking faults calculated from first principles, Acta Materialia 102 (2016) 304-314.

[41] J. A. Yasi, L. G. Hector Jr., D. R. Trinkle, Prediction of thermal cross-slip stress in magnesium alloys from direct first-principles data, Acta Materialia 59 (2011) 5652-5660.

[42] N. E. Paton, W. A. Backofen, Plastic deformation of titanium at elevated temperatures, Metallurgical Transactions 1 (1970) 2839-2847.

[43] A. A. Pochettino, N. Gannio, C. V. Edwards, R. Penelle, Texture and pyramidal slip in $\mathrm{Ti}, \mathrm{Zr}$ and their alloys, Scripta Metallurgica et Materialia 27 (1992) 1859-1863.

[44] S. Zaefferer, A study of active deformation systems in titanium alloys: dependence on alloy composition and correlation with deformation texture, Materials Science and Engineering: A 344 (2003) 20-30.

[45] J. Gong, A. J. Wilkinson, Anisotropy in the plastic flow properties of singlecrystal $\alpha$-titanium determined from micro-cantilever beams, Acta Materialia 57 (2009) 5693-5705. 
[46] L. Wang, R. Barabash, T. Bieler, W. Liu, P. Eisenlohr, Study of $\{11 \overline{2} 1\}$ twinning in $\alpha$-Ti by EBSD and Laue microdiffraction, Metallurgical and Materials Transactions A-Physical Metallurgy and Materials Science 44 (2013) 3664-3674.

[47] A. Akhtar, Compression of zirconium single crystals parallel to the c-axis, Journal of Nuclear Materials 47 (1973) 79-86.

[48] J. Gong, T. Benjamin Britton, M. A. Cuddihy, F. P. E. Dunne, A. J. Wilkinson, $\langle a\rangle$ prismatic, $\langle a\rangle$ basal, and $\langle c+a\rangle$ slip strengths of commercially pure $\mathrm{Zr}$ by micro-cantilever tests, Acta Materialia 96 (2015) 249-257.

[49] R. L. Bell, R. W. Cahn, The dynamics of twinning and the interrelation of slip and twinning in zinc crystals, Proceedings of the Royal Society of London A: Mathematical, Physical and Engineering Sciences 239 (1957) 494-521.

[50] P. B. Price, Pyramidal glide and the formation and climb of dislocation loops in nearly perfect zinc crystals, Philosophical Magazine 5 (1960) 873-886.

[51] P. B. Price, Nonbasal glide in dislocation-free Cadmium crystals. II. the $(1 \overline{1} 2)$ [ $\overline{1} \overline{1} 23]$ system, Journal of Applied Physics 32 (1961) 1750-1757.

[52] H. Tonda, S. Ando, Effect of temperature and shear direction on yield stress by $\{11 \overline{2} 2\}\langle\overline{1} \overline{1} 23\rangle$ slip in HCP metals, Metallurgical and Materials Transactions A - Physical Metallurgy and Materials Science 33 (2002) 831-836.

[53] J. Krásová, P. Kratochvíl, Temperature dependence of the flow stress in secondary glide planes of cadmium single crystals, Physica Status Solidi (a) 7 (1971) 255-261.

[54] P. B. Legrand, Relations entre la structure électronique et la facilitéde glissement dans les métaux hexagonaux compacts, Philosophical Magazine Part B 49 (1984) 171-184.

[55] M. H. Yoo, C. T. Wei, Slip modes of hexagonal-close-packed metals, Journal of Applied Physics 38 (1967) 4317-4322.

[56] S. R. Agnew, J. A. Horton, M. H. Yoo, Transmission electron microscopy investigation of $\langle c+a\rangle$ dislocations in $\mathrm{Mg}$ and $\alpha$-solid solution $\mathrm{Mg}$-Li alloys, Metallurgical and Materials Transactions A 33 (2002) 851-858. 
[57] M. H. Yoo, c + a dislocation reactions in H.C.P. metals, Scripta Metallurgica 2 (1968) 537-540.

[58] Y. T. Chou, Interaction of parallel dislocations in a hexagonal crystal, Journal of Applied Physics 33 (1962) 2747-2751.

[59] M. M. Savin, V. M. Chernov, A. M. Strokova, Energy factor of dislocations in hexagonal crystals, Physica Status Solidi (a) 35 (1976) 747-754.

[60] J. F. Stohr, J. P. Poirier, Etude en microscopie electronique du glissement pyramidal $\{11 \overline{2} 2\}\langle 11 \overline{2} 3\rangle$ dans le magnesium, Philosophical Magazine 25 (1972) 1313-1329.

[61] T. Obara, H. Yoshinga, S. Morozumi, $\{11 \overline{2} 2\}\langle\overline{1} \overline{1} 23\rangle$ slip system in magnesium, Acta Metallurgica 21 (1973) 845-853.

[62] Y. Minonishi, S. Morozumi, H. Yoshinaga, $\{11 \overline{2} 2\}\langle\overline{1} \overline{1} 23\rangle$ slip in titanium, Scripta Metallurgica 16 (1982) 427-430.

[63] S. Sandlöbes, M. Friák, S. Zaefferer, A. Dick, S. Yi, D. Letzig, Z. Pei, L. F. Zhu, J. Neugebauer, D. Raabe, The relation between ductility and stacking fault energies in Mg and Mg-Y alloys, Acta Materialia 60 (2012) 30113021.

[64] S. Sandlöbes, Z. Pei, M. Friák, L. F. Zhu, F. Wang, S. Zaefferer, D. Raabe, J. Neugebauer, Ductility improvement of $\mathrm{Mg}$ alloys by solid solution: $\mathrm{Ab}$ initio modeling, synthesis and mechanical properties, Acta Materialia 70 (2014) 92-104.

[65] J. Geng, M. F. Chisholm, R. K. Mishra, K. S. Kumar, An electron microscopy study of dislocation structures in mg single crystals compressed along [0001] at room temperature, Philosophical Magazine 95 (2015) 39103932.

[66] J. Geng, M. F. Chisholm, R. K. Mishra, K. S. Kumar, The structure of $\langle c+a\rangle$ type dislocation loops in magnesium, Philosophical Magazine Letters 94 (2014) 377-386.

[67] S. Ando, A. Kodera, K. Fukushima, M. Tsushida, H. Kitahara, Tensile deformation of magnesium and magnesium alloy single crystals, Materials Science Forum 783-786 (2014) 341-345. 
[68] I. P. Jones, W. B. Hutchinson, Stress-state dependence of slip in Titanium6Al-4V and other H.C.P. metals, Acta Metallurgica 29 (1981) 951-968.

[69] Y. Minonishi, S. Morozumi, H. Yoshinaga, Accommodation around $\{10 \overline{1} 1\}$ twins in titanium, Scripta Metallurgica 19 (1985) 1241-1245.

[70] J. Kwon, M. C. Brandes, P. Sudharshan Phani, A. P. Pilchak, Y. F. Gao, E. P. George, G. M. Pharr, M. J. Mills, Characterization of deformation anisotropies in an $\alpha$-Ti alloy by nanoindentation and electron microscopy, Acta Materialia 61 (2013) 4743-4756.

[71] X. Feaugas, M. Clavel, Cyclic deformation behaviour of an $\alpha / \beta$ titanium alloy -I. micromechanisms of plasticity under various loading paths, Acta Materialia 45 (1997) 2685-2701.

[72] M. Ghazisaeidi, D. R. Trinkle, Core structure of a screw dislocation in Ti from density functional theory and classical potentials, Acta Materialia 60 (2012) 1287-1292. 

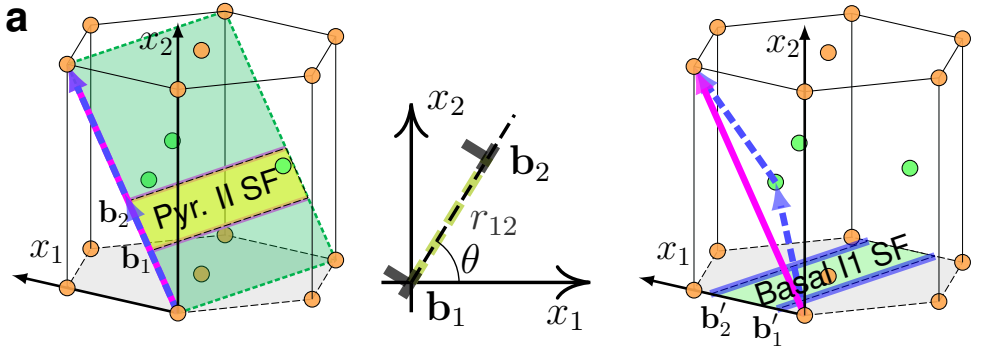

*Graphical Abstract

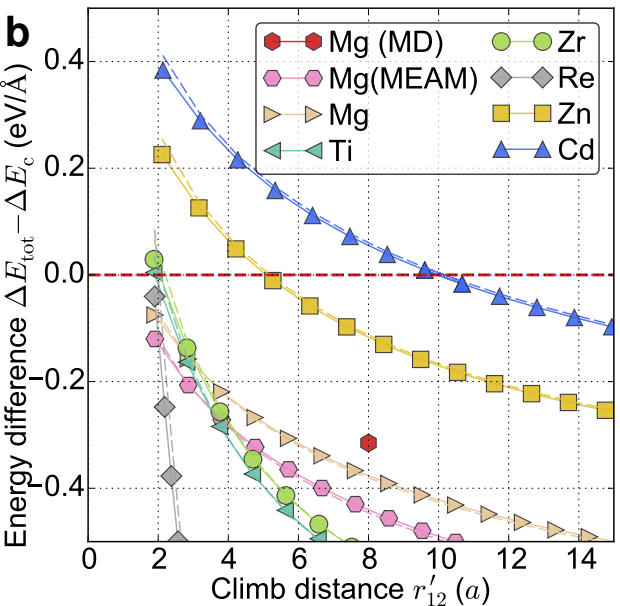

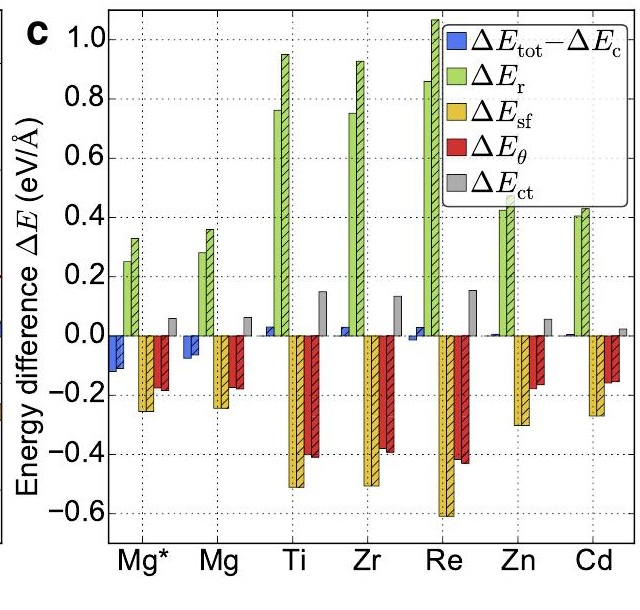

